Fluctuating Nonlinear Oscillators, Ed. Mark I. Dykman

(Oxford University Press, Oxford, 2012), Chapter 11:

\title{
Collective Dynamics in Arrays of Coupled Nonlinear Resonators
}

\author{
Ron Lifshitz \\ Raymond and Beverly Sackler School of Physics 83 Astronomy, Tel Aviv University, Tel Aviv \\ 69978, Israel \\ Eyal Kenig and M.C. Cross \\ Condensed Matter Physics 149-33, California Institute of Technology, Pasadena, CA 91125, \\ USA
}





\section{Contents}

11 Collective Dynamics in Arrays of Coupled Nonlinear Resonators

Ron Lifshitz, Eyal Kenig, and M.C. Cross 1

11.1 Arrays of Nonlinear MEMS \& NEMS Resonators 1

11.2 Equations of Motion and Basic Assumptions 2

11.2.1 Modeling a single nonlinear resonator 3

11.2.2 Modeling an array of nonlinear resonators 3

11.3 Discrete Amplitude Equations:

Example I - Collective response to parametric excitation

11.3.1 Deriving the equations

11.3.2 Analyzing and solving the equations 8

11.3.3 Brief survey of applications 9

11.4 Discrete Amplitude Equations:

Example II - Synchronization of nonlinear oscillators

11.4.1 Deriving the equations

11.4.2 Analyzing and solving the equations 12

11.4.3 Brief survey of applications 14

11.5 Continuous Amplitude Equations:
Example III - Nonlinear competition between extended modes

11.5.1 Derivation of the BCL amplitude equation 15

11.5.2 Analyzing and solving the equation 19

11.5.3 Brief survey of applications 21

11.6 Continuous Amplitude Equations:

Example IV - Intrinsic localized modes (ILMs) 24

11.6.1 Derivation of the PDNLS equation 24

11.6.2 Analyzing and solving the equation 27

11.6.3 Brief survey of applications 29

References 33

Index 37 


\title{
11
}

\section{Collective Dynamics in Arrays of Coupled Nonlinear Resonators}

\author{
Ron Lifshitz, Eyal Kenig, and M.C. Cross
}

\subsection{Arrays of Nonlinear MEMS \& NEMS Resonators}

The study of collective nonlinear dynamics of coupled mechanical resonators is regaining attention in recent years thanks to rapid developments in the fields of microelectromechanical and nanoelectromechanical systems (MEMS and NEMS) (Roukes, 2001; Cleland, 2003). MEMS \& NEMS resonators are typically characterized by very high frequencies, extremely small masses, and weak damping. As such, they are naturally being developed for a variety of applications such as sensing with unprecedented accuracy (Rugar et al., 2004, Ilic et al., 2004, Ekinci et al., 2004, Yang et al., 2006, Li et al., 2007|| Naik et al., 2009; Lee et al., 2010). NEMS, in particular, are being developed also for studying fundamental physics at small scales - exploring mesoscopic phenomena (Schwab et al., 2000; Weig et al., 2004), and even approaching quantum behavior (LaHaye et al., 2004| Naik et al., 2006:|Rocheleau et al., 2010; O'Connell et al., 2010). MEMS \& NEMS resonators often exhibit nonlinear behavior in their dynamics, as recently reviewed by Lifshitz and Cross $(2008,2010)$ and by Rhoads et al. (2010). This includes nonlinear resonant response showing frequency pulling, multistability, and hysteresis (Craighead, 2000; Turner et al., 1998; Zaitsev et al., 2005; Aldridge and Cleland, 2005 (Kozinsky et al., 2007), the appearance of chaotic dynamics (Scheible et al., 2002; DeMartini et al., 2007; Karabalin et al., 2009, Kenig et al., 2011), as well as the formation of extended (Buks and Roukes, 2002) and localized (Sato et al., 2006; Sato and Sievers, 2007, Sato and Sievers, 2008) collective states in arrays of coupled nonlinear resonators. Nonlinearities may be a nuisance in actual applications, and schemes are being developed to avoid them, as demonstrated, for example, by Kacem et al. (2009, 2010). On the other hand, one can also benefit from the existence of nonlinearity, for example in mass-sensing applications (Zhang et al., 2002,Buks and Yurke, 2006), in suppressing noise induced phase diffusion, as suggested by Greywall et al. (1994), and in achieving self-synchronization of large arrays, as proposed by Cross et al. (2004, 2006). Nonlinearity is even proposed by Katz et al. (2007, 2008) as a way to detect quantum behavior in large mechanical systems.

Current technology enables the fabrication of large arrays, composed of hundreds to tens of thousands of MEMS and NEMS devices, coupled by electric, magnetic, or elastic forces. These arrays offer new possibilities for quantitative studies of nonlinear 


\section{Collective Dynamics in Arrays of Coupled Nonlinear Resonators}

dynamics in systems with an intermediate number of degrees of freedom-much larger than one can deal with in macroscopic experiments, yet much smaller than one confronts when considering nonlinear aspects of phonon dynamics in a crystal. Our studies of collective nonlinear dynamics of MEMS and NEMS were originally motivated by the experiment of Buks and Roukes (2002). These studies have led to a quantitative understanding of the collective response of arrays of nonlinear resonators, providing explicit bifurcation diagrams that explain the transitions between different extended modes of an array as the strength and frequency of the external drive are varied quasistatically (Lifshitz and Cross, 2003, Bromberg et al., 2006). We have considered more general issues such as the nonlinear competition between extended modes, or patterns, of the system - when many such patterns are simultaneously stable - as the external driving parameters are changed abruptly or ramped as a function of time (Kenig et al., 2009a). We have also studied the formation, stability, and rich dynamics of intrinsically localized modes (Kenig et al., 2009b). Furthermore, we have investigated the synchronization that may occur in coupled arrays of non-identical nonlinear oscillators, based on the ability of nonlinear oscillators to tune their frequency by changing their oscillation amplitude (Cross et al., 2004, Cross et al., 2006).

The purpose of this chapter is to provide a review of the collective dynamical phenomena observed in these different systems, while highlighting the common concepts and theoretical tools that we have developed for dealing with them. We assume that the reader is familiar with the basic dynamical phenomena associated with single nonlinear resonators. The unfamiliar reader is encouraged to consult our previous review on the subject (Lifshitz and Cross, 2008), or its revised version (Lifshitz and Cross, 2010). In Sec. 11.2 we describe the equations of motion that are used to model arrays of nonlinear MEMS and NEMS resonators, for different experimental realizations. We then give two examples of the derivation and then application of discrete amplitude equations for treating arrays of resonators - in Sec. 11.3 we study the resonant nonlinear response of arrays to parametric excitation, and in Sec. 11.4 we discuss the question of synchronization. We conclude with two examples of the derivation and then application of continuous amplitude equations for treating large arrays of resonators - in Sec. 11.5 for investigating pattern selection, and in Sec. 11.6 for the study of intrinsically localized modes. In place of a formal concluding section, we wish to emphasize at the outset that all the results obtained from analyzing the different amplitude equations are in excellent agreement with numerical solutions of the underlying equations of motion. This upholds the validity of using such reduced descriptions for complex systems, whose original description is given in terms of coupled nonlinear ordinary differential equations. Furthermore, our numerical simulations of the equations of motion suggest that the predicted effects can be observed in arrays of real MEMS and NEMS resonators, thus motivating new experiments in these systems.

\subsection{Equations of Motion and Basic Assumptions}

Typical MEMS and NEMS resonators are characterized by extremely high frequencies - now going beyond $10 \mathrm{GHz}$ (Huang et al., 2003: Cleland and Geller, 2004; Weinstein and Bhave, 2010) - and relatively weak dissipation, with quality factors $Q$ in the range of $10^{2}-10^{5}$. For such devices, under external driving conditions, transients die 
out rapidly, making it is easy to acquire sufficient data to characterize the steady-state well. This, and the fact that weak dissipation and weak nonlinearity can be treated perturbatively, are a great advantage for quantitative comparison between theory and experiment.

\subsubsection{Modeling a single nonlinear resonator}

A typical single resonator is described after appropriate scaling by a dimensionless equation of motion of the form (Lifshitz and Cross, 2008)

$$
\ddot{x}+Q^{-1} \dot{x}+\left[1+H \cos \omega_{P} t\right] x+x^{3}+\eta x^{2} \dot{x}=G \cos \left(\omega_{D} t+\phi_{g}\right) .
$$

We typically use the fact that damping $Q^{-1}$ is much smaller than the resonant frequency, which has been scaled here to 1 , to define the small expansion parameter $\epsilon=Q^{-1}$. The term proportional to $H$ on the left hand side is an external drive that modulates the spring constant. This is a parametric drive - a term that is proportional to the displacement $x$ as well as to the strength of the drive. The term proportional to $G$ on the right-hand side is the standard direct drive, possibly shifted by a phase $\phi_{g}$ with respect to the parametric drive. The coefficient of the nonlinear $x^{3}$ Duffing term has been scaled to 1, and a nonlinear damping term [Dykman and Krivoglaz 1975 , 1984)] with coefficient $\eta$ is also added. For parametric drive, we normally consider the largest excitation effect that occurs when the pump frequency $\omega_{P}$ is close to twice the resonant frequency of the resonator. We therefore take $\omega_{p}=2+\epsilon \Omega_{P}$, and take the drive amplitude to scale as the damping by setting $H=\epsilon h$. The amplitude of the direct drive is scaled as $G=\epsilon^{3 / 2} g$, and its frequency is set an amount $\epsilon \Omega_{D}$ away from the resonant frequency.

The scaled equation of motion that we then obtain is of the form

$$
\ddot{x}+\epsilon \dot{x}+\left(1+\epsilon h \cos \left[\left(2+\epsilon \Omega_{P}\right) t\right]\right) x+x^{3}+\eta x^{2} \dot{x}=\epsilon^{3 / 2}|g| \cos \left[\left(1+\epsilon \Omega_{D}\right) t+\phi_{g}\right],
$$

where we use $g=|g| e^{i \phi_{g}}$ to denote a complex drive amplitude. Ignoring transients, which as explained above decay very rapidly, the solutions to such equations of motion are of the form $x=\sqrt{\epsilon} \Re\left\{A(T) e^{i t}\right\}$ plus corrections of higher order in $\epsilon$, where secular perturbation theory is used to yield the equation that governs the slow dynamics of the complex amplitude $A(T)$. The variable $T=\epsilon t$ is the slow time scale upon which the interesting nonlinear dynamics takes place. Please refer to Lifshitz and Cross (2008, 2010 ) for more details and for many examples of the use of this approach. We only wish to remind the reader that additional nonlinear terms, up to third order in $x$ or $\dot{x}$ such as $x^{2}$ and $x \dot{x}^{2}$, that seem to be missing in eqn 11.2 , merely conspire to renormalize the effective parameters in the slow equation for $A(T)$, but do not affect the actual form of this equation. We therefore ignore all such terms as they have no effect on the actual nature of the solutions that we study.

\subsubsection{Modeling an array of nonlinear resonators}

Lifshitz and Cross (2003) had originally modeled a 1-dimensional array of parametrically driven coupled nonlinear resonators, motivated by the particular experiment of Buks and Roukes (2002), in which an array of 67 doubly-clamped micromechanical 


\section{Collective Dynamics in Arrays of Coupled Nonlinear Resonators}

gold beams was parametrically excited by modulating the strength of an externallycontrolled electrostatic coupling between neighboring beams. We used a set of coupled equations of motion of the form

$$
\begin{gathered}
\ddot{u}_{n}+u_{n}-\frac{1}{2} Q^{-1}\left(\dot{u}_{n+1}-2 \dot{u}_{n}+\dot{u}_{n-1}\right)+\frac{1}{2}\left(D+H \cos \omega_{p} t\right)\left(u_{n+1}-2 u_{n}+u_{n-1}\right) \\
+u_{n}^{3}-\frac{1}{2} \eta\left[\left(u_{n+1}-u_{n}\right)^{2}\left(\dot{u}_{n+1}-\dot{u}_{n}\right)-\left(u_{n}-u_{n-1}\right)^{2}\left(\dot{u}_{n}-\dot{u}_{n-1}\right)\right]=0,
\end{gathered}
$$

where $u_{n}(t)$ describes the deviation of the $n^{t h}$ resonator from its equilibrium, with $n=1 \ldots N$, and fixed boundary conditions $u_{0}=u_{N+1}=0$. Detailed arguments for the choice of terms introduced into these particular equations of motion are discussed by Lifshitz and Cross (2003). We only note that they contain nearest-neighbor linear coupling which is both reactive, proportional to the relative displacements, and dissipative, proportional to the relative velocities; as well as nonlinear dissipative coupling, proportional to the square of the relative displacements and to the relative velocities.

A simpler model, suitable in many other situations, is to take the equation of motion of each resonator to be as in (11.1) with the addition of only a linear reactive coupling term to its two neighbors. The equations of motion then take the form

$$
\ddot{u}_{n}+Q^{-1} \dot{u}_{n}+\left(1+H \cos \omega_{P} t\right) u_{n}+u_{n}^{3}+\eta u_{n}^{2} \dot{u}_{n}+\frac{1}{2} D\left(u_{n+1}-2 u_{n}+u_{n-1}\right)=0,
$$

where in both cases one could add the direct drive, proportional to $G$, that was considered earlier in eqn (11.1).

Finally, to model an array of oscillators - having a frequency-independent source of energy that sustains their oscillations - rather than simple resonators that respond resonantly to an external frequency-dependent drive, we consider a slight modification of eqn 11.4, given by

$$
\ddot{u}_{n}+\omega_{n}^{2} u_{n}-\nu\left(1-u_{n}^{2}\right) \dot{u}_{n}+a u_{n}^{3}+\frac{1}{2} D\left(u_{n+1}-2 u_{n}+u_{n-1}\right)=0 .
$$

In this case both the parametric drive and the direct drive are omitted. Instead, we introduce a negative linear damping with coefficient $\nu$, which represents an energy source to sustain the oscillations, while keeping the positive nonlinear damping so that the oscillation amplitude saturates at a finite value. We use a different scaling than before to set this saturation value to be of order unity, and therefore must reintroduce an explicit coefficient $a$ in front of the $x^{3}$ Duffing term, which can no longer be scaled to unity. One can implement such an effect with an electronic feedback loop, sensing each oscillator velocity and driving the oscillator with an appropriate phase (Feng et al., 2008). The first three terms of eqn (11.5) comprise a so-called van der Pohl oscillator. Note that in anticipation of our study of synchronization of coupled oscillators in Sec. 11.4 below, we have assumed that the uncoupled oscillators can generally have non-identical linear frequencies $\omega_{n}$.

The equations of motion for particular experimental implementations might have different terms, although we expect all will have positive or negative Duffing terms; linear and possibly also nonlinear damping; linear and possibly also nonlinear coupling, which may be either reactive or dissipative; and some source of energy to sustain the oscillations. In many cases, although not always, once we transform to the reduced description describing the slow modulation of the modes (see below), the differences between these different models will not lead to qualitatively new effects. 


\subsection{Discrete Amplitude Equations: Example I - Collective response to parametric excitation}

\subsubsection{Deriving the equations}

As in the case of a single resonator in eqn 11.2, we suppose $Q$ is large and take $\epsilon=Q^{-1}$ as a small expansion parameter. Again, we take $H=\epsilon h$, and in addition also take $D=\epsilon d$ so that the width of the frequency band of normal modes is also small. This is not quite how Lifshitz and Cross (2003) treated the coupling, but it is simpler yet equivalent up to the order of the expansion in $\epsilon$ that we require. The equations of motion (11.4) then become

$\ddot{u}_{n}+\epsilon \dot{u}_{n}+\left(1+\epsilon h \cos \left[\left(2+\epsilon \Omega_{P}\right) t\right]\right) u_{n}+\frac{1}{2} \epsilon d\left(u_{n+1}-2 u_{n}+u_{n-1}\right)+u_{n}^{3}+\eta u_{n}^{2} \dot{u}_{n}=0$.

We expand $u_{n}(t)$ as a sum of standing wave modes with slowly varying amplitudes. The nature of the standing wave modes will depend on the conditions at the ends of the array of resonators. In the experiment of Buks and Roukes (2002) there where $N$ mobile beams with a number of identical immobilized beams at each end. These conditions can be implemented in a nearest neighbor model by taking two additional resonators, $u_{0}$ and $u_{N+1}$ and assuming

$$
u_{0}=u_{N+1}=0 \text {. }
$$

The standing wave modes are then

$$
u_{n}=\sin \left(n q_{m}\right) \quad \text { with } \quad q_{m}=\frac{m \pi}{N+1}, m=1 \ldots N
$$

On the other hand, for an array of $N$ resonators with free ends there is no force from outside the array. For the nearest neighbor model this can be imposed again by taking two additional resonators, but now with the conditions

$$
u_{0}=u_{1} ; \quad u_{N}=u_{N+1}
$$

The standing wave modes are now

$$
u_{n}=\cos \left[\left(n-\frac{1}{2}\right) q_{m}\right] \quad \text { with } \quad q_{m}=\frac{m \pi}{N}, m=0 \ldots N-1
$$

For our illustration we will take eqns 11.7, 11.8.

To treat the equations of motion (11.6) analytically, we use secular perturbation theory combined with a multiple scales analysis, taking advantage of the natural separation of time scales that occurs in our physical system - the fast oscillations of the resonators at half the drive frequency are characterized by the fast time variable $t$, whereas the slow variation of the amplitudes of these oscillations is associated with transient times, characterized by the damping rate $Q^{-1}$, or $\epsilon$, giving rise to a wellseparated slow time variable $T=\epsilon t$. This approach is used throughout this review, 


\section{Collective Dynamics in Arrays of Coupled Nonlinear Resonators}

and was described in great detail in our previous review (Lifshitz and Cross, 2008 Lifshitz and Cross, 2010). Thus, we introduce the ansatz

$$
u_{n}(t)=\epsilon^{1 / 2} \frac{1}{2} \sum_{m=1}^{N}\left(A_{m}(T) \sin \left(n q_{m}\right) e^{i t}+\text { c.c. }\right)+\epsilon^{3 / 2} u_{n}^{(1)}(t)+\ldots, n=1 \ldots N,
$$

where c.c. stands for the complex conjugate. The lowest order contribution to this solution is based on the normal mode solutions 11.8 of the linear equations of motion, allowing the complex mode amplitudes $A_{m}(T)$ to vary slowly in time (as in a rotating frame in the complex plane), due to the effect of all the other terms in the equation. As we shall immediately see, the slow temporal variation of $A_{m}(T)$ also allows us to ensure that the perturbative correction $u_{n}^{(1)}(t)$, as well as all higher-order corrections to the solution (11.11), do not diverge as they do if one uses naive perturbation theory.

Using the relation

$$
\dot{A}_{n}=\frac{d A_{n}}{d t}=\epsilon \frac{d A_{n}}{d T} \equiv \epsilon A_{n}^{\prime},
$$

and denoting a time derivative with respect to the slow time $T$ by a prime, we substitute the trial solution (11.11) into the equations of motion (11.6) term by term. Up to order $\epsilon^{3 / 2}$ we have,

$$
\begin{aligned}
& \ddot{u}_{n}=\epsilon^{1 / 2} \frac{1}{2} \sum_{m} \sin \left(n q_{m}\right)\left(\left[-A_{m}+2 i \epsilon A_{m}^{\prime}\right] e^{i t}+c . c .\right)+\epsilon^{3 / 2} \ddot{u}_{n}^{(1)}(t), \\
& \epsilon \dot{u}_{n}=\epsilon^{3 / 2} \frac{1}{2} \sum_{m} \sin \left(n q_{m}\right)\left(i A_{m} e^{i t}+c . c .\right) \\
& \epsilon \frac{d}{2}\left(u_{n+1}-2 u_{n}+u_{n-1}\right)=-\epsilon^{3 / 2} \frac{d}{2} \sum_{m} 2 \sin ^{2}\left(\frac{q_{m}}{2}\right) \sin \left(n q_{m}\right)\left(A_{m} e^{i t}+c . c .\right), \\
& u_{n}^{3}=\epsilon^{3 / 2} \frac{1}{8} \sum_{j, k, l} \sin \left(n q_{j}\right) \sin \left(n q_{k}\right) \sin \left(n q_{l}\right)\left(A_{j} e^{i t}+c . c .\right)\left(A_{k} e^{i t}+c . c .\right)\left(A_{l} e^{i t}+\text { c.c. }\right) \\
& =\epsilon^{3 / 2} \frac{1}{32} \sum_{j, k, l}\left\{\sin \left[n\left(-q_{j}+q_{k}+q_{l}\right)\right]+\sin \left[n\left(q_{j}-q_{k}+q_{l}\right)\right]+\sin \left[n\left(q_{j}+q_{k}-q_{l}\right)\right]\right. \\
& \left.\quad-\sin \left[n\left(q_{j}+q_{k}+q_{l}\right)\right]\right\}\left\{A_{j} A_{k} A_{l} e^{3 i t}+3 A_{j} A_{k} A_{l}^{*} e^{i t}+c . c .\right\}
\end{aligned}
$$

and

$$
\begin{aligned}
\eta u_{n}{ }^{2} \dot{u}_{n} & =\epsilon^{3 / 2} \frac{\eta}{32} \sum_{j, k, l}\left\{\sin \left[n\left(-q_{j}+q_{k}+q_{l}\right)\right]+\sin \left[n\left(q_{j}-q_{k}+q_{l}\right)\right]+\sin \left[n\left(q_{j}+q_{k}-q_{l}\right)\right]\right. \\
& \left.-\sin \left[n\left(q_{j}+q_{k}+q_{l}\right)\right]\right\}\left(A_{j} e^{i t}+\text { c.c. }\right)\left(A_{k} e^{i t}+\text { c.c. }\right)\left(i A_{l} e^{i t}+\text { c.c. }\right) . \quad(11.13 \mathrm{e})
\end{aligned}
$$

The order $\epsilon^{1 / 2}$ terms cancel, and at order $\epsilon^{3 / 2}$ we get $N$ equations of the form

$$
\ddot{u}_{n}^{(1)}+u_{n}^{(1)}=\sum_{m}\left(m^{t h} \text { secular term }\right) e^{i t}+\text { other terms, }
$$

where the left-hand sides are uncoupled linear harmonic resonators, with a frequency unity. On the right-hand sides we have $N$ secular terms which act to drive the resonators $u_{n}^{(1)}$ at their resonance frequencies. As Lifshitz and Cross 2008,2010$)$ did for 
all their single resonator examples, here too we require that all the secular terms vanish so that the $u_{n}^{(1)}$ remain finite. This is the necessary solvability condition, required to obtain equations for the slowly varying amplitudes $A_{m}(T)$. To extract the equation for the $m^{t h}$ amplitude $A_{m}(T)$ we make use of the orthogonality of the modes, multiplying all the terms by $\sin \left(n q_{m}\right)$ and summing over $n$. We find that the coefficient of the $m^{\text {th }}$ secular term, which is required to vanish, is given by

$$
-2 i \frac{d A_{m}}{d T}-i A_{m}+2 d \sin ^{2}\left(\frac{q_{m}}{2}\right) A_{m}-\frac{1}{2} h A_{m}^{*} e^{i \Omega_{P} T}-\frac{3+i \eta}{16} \sum_{j, k, l} A_{j} A_{k} A_{l}^{*} \Delta_{j k l ; m}^{(1)}=0,
$$

where we have used the $\Delta$ function introduced by Lifshitz and Cross (2003), defined in terms of Kronecker deltas as

$$
\begin{aligned}
\Delta_{j k l ; m}^{(1)} & =\delta_{-j+k+l, m}-\delta_{-j+k+l,-m}-\delta_{-j+k+l, 2(N+1)-m} \\
& +\delta_{j-k+l, m}-\delta_{j-k+l,-m}-\delta_{j-k+l, 2(N+1)-m} \\
& +\delta_{j+k-l, m}-\delta_{j+k-l,-m}-\delta_{j+k-l, 2(N+1)-m} \\
& -\delta_{j+k+l, m}+\delta_{j+k+l, 2(N+1)-m}-\delta_{j+k+l, 2(N+1)+m}
\end{aligned}
$$

and have exploited the fact that it is invariant under any permutation of the indices $j$, $k$, and $l$. The $\Delta$ function ensures the conservation of lattice momentum - the conservation of momentum to within the non-uniqueness of the specification of the normal modes due to the fact that $\sin \left(n q_{m}\right)=\sin \left(n q_{2 k(N+1) \pm m}\right)$ for any integer $k$. The first Kronecker delta in each line is a condition of direct momentum conservation, and the other two are the so-called umklapp conditions where only lattice momentum is conserved.

As for the single resonator (Lifshitz and Cross, 2008), we again try a steady-state solution, this time of the form

$$
A_{m}(T)=a_{m} e^{i\left(\frac{\Omega_{P}}{2}\right) T},
$$

so that the solutions to the equations of motion (11.6), after substitution of (11.17) into (11.11, become

$$
u_{n}(t)=\epsilon^{1 / 2} \frac{1}{2} \sum_{m}\left(a_{m} \sin \left(n q_{m}\right) e^{i\left(1+\frac{\epsilon \Omega_{P}}{2}\right) t}+c . c .\right)+O\left(\epsilon^{3 / 2}\right),
$$

where all modes are oscillating at half the parametric excitation frequency, $\omega_{P}=$ $2+\epsilon \Omega_{P}$.

Substituting the steady state solution 11.17 into the equations 11.15 for the time-varying amplitudes $A_{m}(T)$, we obtain the equations for the time-independent complex amplitudes $a_{m}$

$$
\left[\Omega_{P}+2 d \sin ^{2}\left(\frac{q_{m}}{2}\right)-i\right] a_{m}-\frac{h}{2} a_{m}^{*}-\frac{3+i \eta}{16} \sum_{j, k, l} a_{j} a_{k} a_{l}^{*} \Delta_{j k l ; m}^{(1)}=0 .
$$

Note that the first two terms on the left-hand side indicate that the linear resonance frequency is not obtained for $\Omega_{P}=0$, but rather for $\Omega_{P}+2 d \sin ^{2}\left(q_{m} / 2\right)=0$. In terms 


\section{Collective Dynamics in Arrays of Coupled Nonlinear Resonators}

of the unscaled parameters, this implies that the resonance frequency of the $m^{\text {th }}$ mode is $\omega_{m}=1-D \sin ^{2}\left(q_{m} / 2\right)$, which to within a correction of $O\left(\epsilon^{2}\right)$ is the same as the expected dispersion relation

$$
\omega_{m}^{2}=1-2 D \sin ^{2}\left(\frac{q_{m}}{2}\right) .
$$

Equations 11.15 and $(11.19)$ are the main result of the calculation. We have managed to replace $N$ coupled differential equations (11.4) for the resonator coordinates $u_{n}(t)$ by $N$ coupled differential equations (11.15) for the slowly varying mode amplitudes $A_{m}(T)$, and then by $N$ coupled algebraic equations 11.19 for the timeindependent mode amplitudes $a_{m}$. All that remains, in order to obtain the overall collective response of the array as a function of the parameters of the original equations of motion (11.4), is to solve these coupled algebraic equations.

\subsubsection{Analyzing and solving the equations}

A number of simple results can immediately be stated. First, one can easily verify that for a single resonator $(N=j=k=l=m=1)$, the general equation (11.19) reduces to the single-resonator equation treated by Lifshitz and Cross $(2008,2010)$, as $\Delta_{111 ; 1}=4$. Next, one can also see that the trivial solution, $a_{m}=0$ for all $m$, always satisfies the equations, though, as Lifshitz and Cross (2008, 2010) showed in the case of a single resonator, it is not always a stable solution. Finally, one can also verify that whenever for a given $m, \Delta_{m m m ; j}^{(1)}=0$ for all $j \neq m$, then a single-mode solution exists with $a_{m} \neq 0$ and $a_{j}=0$ for all $j \neq m$. These single-mode solutions have the same type of elliptical shape of the single-resonator solution. Note that generically $\Delta_{m m m ; m}^{(1)}=3$, except when umklapp conditions are satisfied.

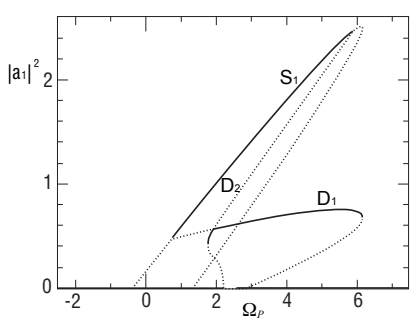

(a)

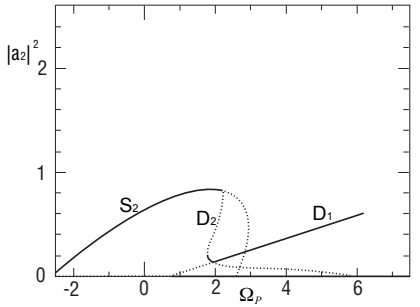

(b)

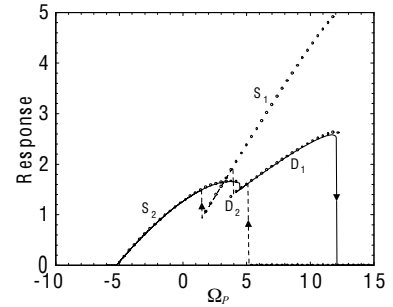

(c)

Fig. 11.1 Response intensity of two resonators as a function of frequency $\Omega_{P}$, for a particular choice of the equation parameters. (a) shows $\left|a_{1}\right|^{2}$, and (b) shows $\left|a_{2}\right|^{2}$, with solid curves indicating stable solutions and dashed curves indicating unstable solutions. (c) Comparison of stable solutions, obtained algebraically (small circles), with a numerical integration of the equations of motion 111.4 (solid curve - frequency swept up; dashed curve - frequency swept down) showing hysteresis in the response. Plotted is the averaged response intensity, defined in the text. In all figures, the two elliptical single-mode solution branches are labeled $S_{1}$ and $S_{2}$, and the two double-mode solution branches are labeled $D_{1}$ and $D_{2}$. From Lifshitz and Cross (2003). Copyright (2003) American Physical Society. 
Additional solutions, involving more than a single mode, exist in general but are hard to obtain analytically. Lifshitz and Cross (2003) calculated these multi-mode solutions explicitly for the case of two and three resonators, for the model they considered, by finding the roots of the coupled algebraic equations numerically. We present some of their results to illustrate the type of behavior that occurs, although the precise details will be slightly different in the model used here. In Fig. 11.1 we show the solutions for the response intensity of two resonators as a function of frequency, for a particular choice of the equation parameters. Figure 11.1(a) shows the square of the amplitude of the symmetric mode $a_{1}$, whereas Fig. 11.1(b) shows the square of the amplitude of the antisymmetric mode $a_{2}$. Solid curves indicate stable solutions and dashed curves indicate unstable solutions. Two elliptical single-mode solution branches, similar to the response of a single resonator are easily spotted. These branches are labeled by $S_{1}$ and $S_{2}$. Lifshitz and Cross (2003) give the analytical expressions for these two solution branches. In addition, there are two double-mode solution branches, labeled $D_{1}$ and $D_{2}$, involving the excitation of both modes simultaneously. Note that the two branches of double-mode solutions intersect at a point where they switch their stability.

With two resonators there are regions in frequency where three stable solutions can exist. If all the stable solution branches are accessible experimentally then the observed effects of hysteresis might be more complex than in the simple case of a single resonator. This is demonstrated in Fig. 11.1(c) where the algebraic solutions are compared with a numerical integration of the differential equations of motion (11.4) for two resonators. The response intensity, plotted here, is the time and space averages of the square of the resonator displacements $\left(\left\langle u_{1}^{2}\right\rangle+\left\langle u_{2}^{2}\right\rangle\right) / 2$, where the angular brackets denote time average. A solid curve shows the response intensity for an upward quasistatic frequency sweep, and a dashed curve shows the response intensity for a downward sweep. Small circles show the response intensity, as calculated for the stable regions of the four algebraic solution branches shown in Figs. 11.1(a) and (b), demonstrating the great utility of the slow amplitude equations. With the analytical solution in the background, one can easily understand all the discontinuous jumps, as well as the hysteresis effects, that are obtained in the numerical solution of the equations of motion. Note that the

$S_{1}$ branch is missed in the upward frequency sweep and is only accessed by the system in the downward sweep. One could trace the whole stable region of the $S_{1}$ branch by changing the sweep direction after jumping onto the branch, thereby climbing all the way up to the end of the $S_{1}$ branch. These kinds of changes in the direction of the quasistatic sweep whenever one jumps onto a new branch are essential if one wants to trace out as much of the solution as possible - whether in real experiments or in numerical simulations.

\subsubsection{Brief survey of applications}

Discrete amplitude equations like the ones derived here (11.19) are useful mainly when studying small arrays. Nevertheless, the insight gained by studying small arrays, of even two or three resonators, provides better understanding of the dynamics of large arrays, which can be studied directly by numerically integrating the starting equations of motion 11.4 . Indeed, all the features of the original experiment of Buks and Roukes (2002) were qualitatively reproduced by Lifshitz and Cross (2003) by numerically 
integrating their original equations of motion (11.3). But, it was their analytical study of small arrays, that allowed them to provide an explanation for the observed features: (1) The response of the system at frequencies above the top edge of the band was attributed to the positive frequency pulling coming from the Duffing nonlinearity; (2) The fact that only a few features are observed in a monotonic quasistatic frequency scan, rather than $N$ resonance peaks for the $N$ normal modes, was explained by the fact that a solution branch is followed quasistatically as long as it is stable, often skipping many other solutions that are simultaneously stable, as demonstrated above with 2 resonators; and (3) The abrupt jumps in the response were identified as stemming from bifurcation points where a certain solution branch ends, as in the saddle-node bifurcation at the end of the $D_{1}$ branch in Fig. 11.1, or simply loses its stability, as for the two $S$ branches in Fig. 11.1, in either case requiring the system to switch abruptly to a different branch.

The curious reader is encouraged to consult additional articles, where the methods presented in this section were used to study advanced features in the dynamics of small numbers of coupled resonators. Karabalin et al. (2009) used discrete amplitude equations for the two normal modes of a pair of resonators, similar to eqn (11.15), to assist in their numerical modeling of period doubling and a transition to chaos, which they observed experimentally. Kenig et al. (2011) used discrete amplitude equations to identify homoclinic orbits in the slow dynamics and assess the possibility of obtaining chaotic dynamics via the Melnikov approach. Finally, Karabalin et al. (2011) demonstrated the use of a pair of parametrically driven resonators as a novel amplifier, whose operation is based on very sensitive control of the bifurcation diagram of the response of two resonators, via an input signal that is fed into the coupling $D$ between the resonators. The Supplementary Material of Karabalin et al. (2011) provides a detailed analysis of the operation of this so-called Bifurcation-Topology Amplifier, using a set of discrete amplitude equations like the ones developed here.

\subsection{Discrete Amplitude Equations: Example II - Synchronization of nonlinear oscillators}

\subsubsection{Deriving the equations}

Although synchronization is often put forward as an example of the importance of understanding nonlinear phenomena, the intuition for it, and indeed the subsequent mathematical discussion, often reduces to simple linear ideas. For example, the famous example of Huygens's clocks (Bennett et al., 2002) can be understood in terms of a linear coupling of the two pendulums through the common mounting support. It is then the larger damping of the symmetric mode (coming from the larger, dissipative motion of the common support) compared with the antisymmetric mode that leads, at long times, to a synchronized state of the two pendulums oscillating in antiphase. The nonlinearity in the system is simply present in the individual motion of each pendulum; specifically in the mechanism to sustain the oscillations. Without the drive, the oscillators would still become synchronized through the faster decay of the even mode, albeit in a slowly decaying state. Rather than this mode-dependent dissipation mechanism, one might expect synchronization to arise from the intrinsically nonlinear 
effect of the frequency pulling of one oscillator by another. Furthermore, the model describing the two Huygens pendulums, as well as most other models used to show synchronization, has dissipative coupling between the oscillators. In contrast, many physical situations have mainly reactive coupling. Consequently, Cross et al. $(2004$, 2006) proposed and analyzed a model for synchronization, given by eqn (11.5), involving reactive coupling between the oscillators, which then leads to synchronization through nonlinear frequency pulling.

We follow Cross et al. (2004, 2006) and consider the system of oscillators defined by eqn (11.5), assuming that the linear frequencies of the oscillators are distributed near unity such that

$$
\omega_{n}^{2}=1+\Delta_{n}, \quad \text { with } \quad\left|\Delta_{n}\right| \ll 1 .
$$

This allows us to study the situation in which the equations of motion are dominated by the terms describing simple harmonic oscillators at frequency one, and the time dependence remains close to $e^{ \pm i t}$. The interesting dynamics should then be captured by a discrete set of coupled amplitude equations for the deviations of the individual oscillators from simple harmonic oscillation at frequency 1 . To that end we assume that all corrections in eqn 11.5 to a set of uncoupled harmonic oscillators of frequency 1 are small. To formalize this smallness we again use the damping term to define a small parameter $\epsilon=\nu$, and take $\Delta_{n}=\epsilon \delta_{n}, a=\epsilon \alpha / 3, D=\epsilon \beta$. The oscillating displacement is then written as a slow modulation of oscillations at frequency one, plus corrections

$$
u_{n}(t)=\left[A_{n}(T) e^{i t}+\text { c.c. }\right]+\epsilon u_{n}^{(1)}(t)+\ldots
$$

with $T=\epsilon t$ a slow time scale as before. As always, the slow variation of $A_{n}(T)$ gives us the extra freedom to eliminate secular terms and ensure that the perturbative correction $u_{n}^{(1)}(t)$, as well as all higher-order corrections to the linear response, do not diverge. Note that our decision to scale eqn 11.5 by setting the van der Pohl term such that the nonlinear saturation of the oscillations occurs at $u_{n}=O(1)$, has affected the scaling of our trial solution (11.22), whose leading term is indeed of order 1. Compare this with the trial solution of the previous section, given by eqn (11.11), whose leading term is of $O(\sqrt{\epsilon})$.

Using the relation 11.12 , again denoting a time derivative with respect to the slow time $T$ by a prime, we calculate the time derivatives of the trial solution 11.22

$$
\begin{aligned}
& \dot{u}_{n}=\left(\left[i A_{n}+\epsilon A_{n}^{\prime}\right] e^{i t}+c . c .\right)+\epsilon \dot{u}_{n}^{(1)}(t)+\ldots \\
& \ddot{u}_{n}=\left(\left[-A_{n}+2 i \epsilon A_{n}^{\prime}+\epsilon^{2} A_{n}^{\prime \prime}\right] e^{i t}+\text { c.c. }\right)+\epsilon \ddot{u}_{n}^{(1)}(t)+\ldots
\end{aligned}
$$

Substituting these expressions back into the scaled equation of motion

$$
\ddot{u}_{n}+\left(1+\epsilon \delta_{n}\right) u_{n}-\epsilon\left[\left(1-u_{n}^{2}\right) \dot{u}_{n}+\frac{1}{3} \alpha u_{n}^{3}+\frac{1}{2} \beta\left(u_{n+1}-2 u_{n}+u_{n-1}\right)\right]=0,
$$

and picking out all terms of order $\epsilon$, we get the following equation for the first perturbative correction

$$
\begin{aligned}
\ddot{u}_{n}^{(1)}+u_{n}^{(1)}= & -\delta_{n} A_{n}-\left(2 i A_{n}^{\prime} e^{i t}+\text { c.c. }\right)+\left(i A_{n} e^{i t}+\text { c.c. }\right)\left[1-\left(A_{n} e^{i t}+\text { c.c. }\right)^{2}\right] \\
& -\frac{1}{3} \alpha\left(A_{n} e^{i t}+\text { c.c. }\right)^{3}+\frac{1}{2} \beta\left[\left(A_{n+1}-2 A_{n}+A_{n-1}\right) e^{i t}+c . c .\right] .
\end{aligned}
$$




\section{Collective Dynamics in Arrays of Coupled Nonlinear Resonators}

Terms varying as $e^{ \pm 3 i t}$ on the right-hand side of Eq. 11.25 contribute a finite response to $u_{n}^{(1)}$, but the collection of terms proportional to $e^{i t}$ - the secular terms - act like a force driving the simple harmonic oscillator on the left-hand side at its resonance frequency. The sum of all these secular terms must vanish so that the perturbative correction $u_{n}^{(1)}(t)$ in eqn 11.22 will not diverge. This provides us with a solvability condition that leads to an equation for determining the slowly varying amplitudes $A_{n}(T)$

$$
2 \frac{d A_{n}}{d T}=\left(1+i \delta_{n}\right) A_{n}-(1-i \alpha)\left|A_{n}\right|^{2} A_{n}+i \frac{\beta}{2}\left(A_{n+1}-2 A_{n}+A_{n-1}\right) .
$$

With a rescaling of time $\tau=T / 2=\epsilon t / 2$ and a slight rearrangement of terms, eqn 11.26 reduces to the form obtained by Cross et al. (2004, 2006),

$$
\frac{d A_{n}}{d \tau}=i\left(\delta_{n}+\alpha\left|A_{n}\right|^{2}\right) A_{n}+\left(1-\left|A_{n}\right|^{2}\right) A_{n}+i \frac{\beta}{2}\left(A_{n+1}-2 A_{n}+A_{n-1}\right)
$$

The first term on the right-hand side shows the ability of the $n^{\text {th }}$ oscillator to shift its frequency by an amount $\alpha\left|A_{n}\right|^{2}$; the second term shows the tendency of the

oscillators to increase their amplitude as long as $\left|A_{n}\right|^{2}<1$; and the third term is the reactive coupling between nearest neighbors. If this nearest-neighbor coupling is generalized to allow also dispersive interaction and replaced by an all-to-all or meanfield coupling, convenient for theoretical analysis, we obtain the final form of the model studied by Cross et al. (2004, 2006),

$$
\frac{d A_{n}}{d \tau}=i\left(\delta_{n}+\alpha\left|A_{n}\right|^{2}\right) A_{n}+\left(1-\left|A_{n}\right|^{2}\right) A_{n}+\frac{K+i \beta}{N} \sum_{m=1}^{N}\left(A_{m}-A_{n}\right),
$$

except for the fact that we use the opposite sign convention for the Duffing parameter. Thus, in our current discussion a positive (negative) value of $\alpha$ implies a stiffening (softening) Duffing nonlinearity. The relative natural frequency $\delta_{n}$ of each oscillator is chosen from a specified distribution $g(\delta)$, whose width is denoted by $w$.

When only nonlinear saturation and dissipative coupling are present $(\alpha=\beta=$ $0, K \neq 0$ ) eqn 11.28 reduces to

$$
\frac{d A_{n}}{d \tau}=\left(i \delta_{n}+1-\left|A_{n}\right|^{2}\right) A_{n}+\frac{K}{N} \sum_{m=1}^{N}\left(A_{m}-A_{n}\right)
$$

which has been analyzed by Matthews et al. (1991) for general $w$ and $K$.

\subsubsection{Analyzing and solving the equations}

The complex number $A_{n}$, representing the amplitude $r_{n}$ and phase $\theta_{n}$ of the $n^{\text {th }}$ oscillator, $A_{n}=r_{n} e^{i \theta_{n}}$, suggests the introduction of a complex order parameter $\Psi$ to measure the coherence of the oscillations 


$$
\Psi=R e^{i \Theta}=\frac{1}{N} \sum_{n=1}^{N} r_{n} e^{i \theta_{n}} .
$$

A nonzero value of the order parameter $R>0$ may be taken as the definition of a synchronized state.

The general amplitude-phase model reduces to familiar phase only models of synchronization in certain limits. If the width $w$ of the distribution $g(\delta)$ is narrow, so that the time evolution of the magnitudes $r_{n}=\left|A_{n}\right|$ is fast compared with that of the phase dispersion, and the coupling constants $K, \beta$ are small, $r_{n}$ rapidly relaxes to a value close to unity

$$
r_{n}^{2} \simeq 1+\frac{K}{N} \sum_{m=1}^{N}\left[\cos \left(\theta_{m}-\theta_{n}\right)-1\right]-\frac{\beta}{N} \sum_{m=1}^{N} \sin \left(\theta_{m}-\theta_{n}\right),
$$

and the only remaining dynamical variable for each oscillator is its phase $\theta_{n}$. Equation (11.28) can then be reduced to

$$
\dot{\theta}_{n}=\delta_{n}+\alpha+\frac{K-\alpha \beta}{N} \sum_{m=1}^{N} \sin \left(\theta_{m}-\theta_{n}\right)+\frac{\alpha K+\beta}{N} \sum_{m=1}^{N}\left[\cos \left(\theta_{m}-\theta_{n}\right)-1\right] .
$$

For the case of purely dissipative coupling $\alpha=\beta=0, K \neq 0$, or reactive coupling with strong frequency pulling $K=0, \alpha, \beta \neq 0,|\alpha| \gg 1$, the last term on the right hand side of eqn $\sqrt{11.32}$ ) can be neglected, and the equation reduces to a simple form (Winfree, 1967; Kuramoto, 1975), known as the Kuramoto model (ignoring the unimportant constant term $\alpha$ and writing the effective coupling constant in either case simply as a $K)$

$$
\dot{\theta}_{n}=\delta_{n}+\frac{K}{N} \sum_{m=1}^{N} \sin \left(\theta_{m}-\theta_{n}\right)
$$

that has been the subject of numerous studies (Acebrón et al., 2005). In the absence of coupling each oscillator in this model would simply advance at a rate that is constant in time, but with some dispersion of frequencies over the different elements.

Identifying the imaginary part of $\Psi e^{-i \theta_{n}}$ in the sum appearing in eqn 11.33 while recalling that $r_{n}=1$ for the Kuramoto model-yields a particularly simple mean-field expression

$$
\dot{\theta}_{n}=\delta_{n}+K R \sin \left(\Theta-\theta_{n}\right) .
$$

Thus the behavior of each oscillator is given by its tendency to lock to the phase of the order parameter. The term $K R \sin \left(\Theta-\theta_{n}\right)$ acts as a locking force, and locking occurs for all oscillators with frequencies satisfying $\left|\delta_{n}\right|<K R$, with the locked oscillator phase given by $\Theta+\sin ^{-1}\left(\delta_{n} / K R\right)$. The magnitude $R$ of the order parameter must then be determined self-consistently via eqn (11.30).

Equation 11.33 is known to show rich behavior, including, in the large $N$ limit, a sharp synchronization transition at some value of the coupling constant $K=K_{c}$ (Kuramoto, 1975), which depends on the frequency distribution $g(\delta)$ of the uncoupled 
oscillators. The transition is from an unsynchronized state with $\Psi=0$ in which the oscillators run at their individual frequencies, to a synchronized state with $\Psi \neq 0$ in which a finite fraction of the oscillators lock to a single frequency. The transition at $K_{c}$ has many of the features of a second order phase transition, with universal power laws and critical slowing down (Kuramoto, 1975), as well as a diverging response to an applied force (Sakaguchi, 1988).

The last term in eqn (11.32) may lead to important qualitative effects even if the coefficient is not very large. For example, in the absence of this term the coupling terms cancel when summing over all the oscillators in the system, so that the frequency of a synchronized state is simply related to the mean frequency of the oscillators. This is no longer the case for the general equation. In the case of short range, rather than all to all coupling, the $\cos \left(\theta_{m}-\theta_{n}\right)$ term profoundly changes the nature of the synchronized state to one of propagating waves (Sakaguchi et al., 1988; Blasius and Tönjes, 2005).

\subsubsection{Brief survey of applications}

The synchronization of oscillators with reactive all-to-all coupling and nonlinear frequency pulling, described by of eqn 11.28 with $K=0$, was analyzed by Cross et al. (2004, 2006) for several different frequency distributions $g(\delta)$ (Lorentzian, top-hat, and triangular). Here we briefly review the results for a triangular distribution with width $w=2$, and refer the reader to the original work for more details. Such a width is not small compared with the relaxation rate of the magnitude variables, and so the behavior is richer than in the weak randomness limit described by the Kuramoto model. The stability diagram of the variety of states found as $\alpha$ and $\beta$ are varied is shown in Fig. 11.2(d). The results are shown for $\alpha \beta<0$, noting that for a symmetric distribution of frequencies the results are the same if both signs of $\alpha$ and $\beta$ are changed. These same results were presented by Cross et al. (2004, 2006) for their case $\alpha \beta>0$, as they were using the opposite sign convention for $\alpha$.

Certain results can be obtained analytically, in particular the instability from the unsynchronized state to a synchronized state with nonzero order parameter $R>0$, and the instability from the fully locked state (all oscillators locked to evolve at the same frequency) to a synchronized state with only partial locking. Other results are obtained numerically by performing sweeps of $\beta$ at fixed values of $\alpha$. Results for the time averaged magnitude of the order parameter $\langle R\rangle_{t}$ for three values of $\alpha$ are shown in Fig. 11.2(a)-(c). Both upward and downward quasistatic sweeps of the reactive coupling strength $\beta$ are used to uncover hysteresis in the transitions.

Many novel features are apparent in these results. For example, the unsynchronized state is stable for both small and large values of the coupling strength $\beta$, so that for fixed $\alpha$ there are two values of $\beta$ at which the unsynchronized state passes from stable to unstable. At large values of $\beta$ a large order-parameter synchronized state is also stable, which becomes the fully locked state at large enough $\beta$. The transition from the unsynchronized state to the synchronized state may be continuous, passing through a supercritical bifurcation, or discontinuous, passing through a subcritical bifurcation. Hysteresis is apparent in the latter case, owing to the bistability of both the synchronized and the unsynchronized states. This is demonstrated explicitly in Fig. 11.2(c). More surprisingly, one also observes the multistability of different synchronized states. 

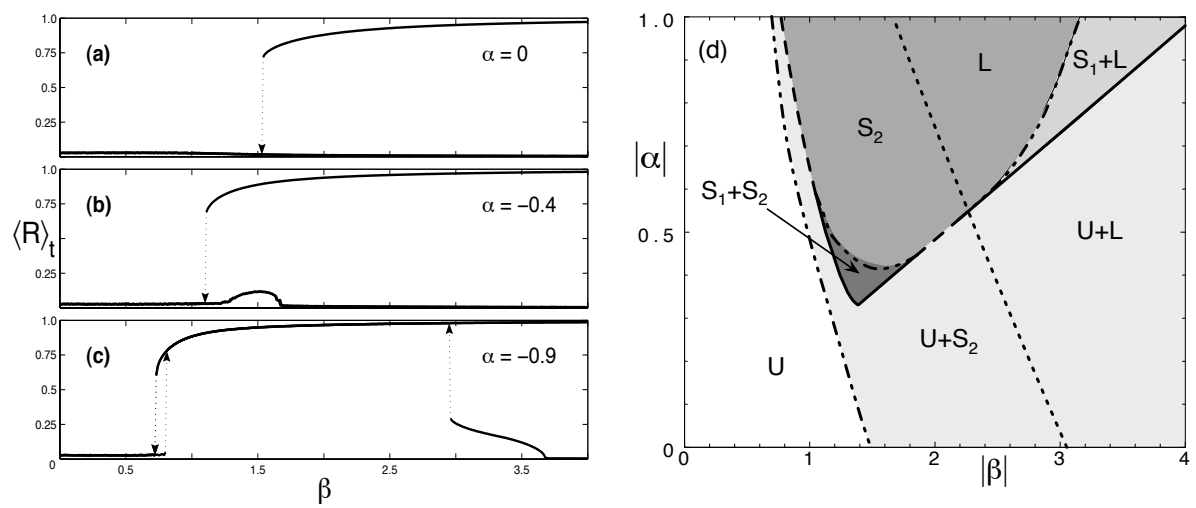

Fig. 11.2 (a)-(c) Simulations of 1000 oscillators having a triangular frequency distribution with width $w=2$. The time-averaged order parameter magnitude $\langle R\rangle_{t}$ is plotted for both upward and downward sweeps of $\beta$ at fixed $\alpha$ : (a) $\alpha=0.0$; (b) $\alpha=-0.4$; and (c) $\alpha=-0.9$. The same results would be obtained by switching the signs of both $\alpha$ and $\beta$. (d) Stability diagram for the same triangular distribution, in the quadrant of the $\alpha-\beta$ plane with $\alpha \beta<0$. Solid and dashed lines show analytical results of the linear stability of the unsynchronized state. Numerics show the bifurcations are supercritical along the solid portions and subcritical along the dashed portion. Dotted line is the linear stability boundary of the fully locked state. Dash-dotted lines are saddle-node bifurcations observed in numerical simulations. States are: $U$ - unsynchronized; $S_{1}, S_{2}$ synchronized with small and large amplitude respectively; $L$ fully locked. From Cross et al. (2006). Copyright (2006) American Physical Society.

Over some parameter ranges a small order-parameter synchronized state may coexist with the large order-parameter synchronized state, as observed, for example, in Fig. 11.2 (c) between about $\beta=3.0$ and $\beta=3.7$. This small order-parameter synchronized state has the novel property that the order parameter is nonzero $R>0$, but there is no oscillator locked in frequency to the frequency of the order parameter or of other oscillators - this is a synchronized state $R>0$ with no frequency locking.

The rich synchronization behavior displayed by this stability diagram opens up many possibilities for applications, as well as suggesting difficulties that must be overcome, for example when there exists a multistability of different dynamical states.

\subsection{Continuous Amplitude Equations: Example III - Nonlinear competition between extended modes}

\subsubsection{Derivation of the BCL amplitude equation}

We wish to investigate the sequence of single mode standing wave patterns to be expected in parametrically driven resonator arrays, in cases where many such modes are simultaneously stable, when the strength of the drive is varied. Although the quantitative analysis could be done directly from the basic equations of motion for the coupled resonators, it is advantageous to formulate the analysis in terms of a continuous amplitude equation - that which was developed by Bromberg, Cross and Lifshitz (2006), 


\section{Collective Dynamics in Arrays of Coupled Nonlinear Resonators}

henceforth referred to as the BCL equation. This allows us to display the range of stable patterns on a reduced stability diagram involving just two dimensionless variables (a scaled measure of the driving strength, and a scaled mode wave number), so that it is easy to deduce the general qualitative behavior upon variation of the parameters. The specific quantitative behavior for a physical system is also easy to obtain by evaluating the corresponding scaled quantities. A change of pattern occurs when parameters vary so that the mode moves outside of the region of stable patterns on this diagram, and the new pattern is predicted by analyzing the result of the instability using the BCL equation. This type of approach was used in other pattern forming systems (Kramer et al., 1988). A novel feature of the present system is that the difference in the instabilities encountered on increasing and decreasing the (scaled) driving strength leads to the prediction of quite different-sized mode jumps for the up and down sweeps.

We follow the treatment of BCL in deriving their amplitude equation, but instead of starting with the original equations of motion 11.3 derived by Lifshitz and Cross (2003), we start with the simpler equations of motion (11.4). This leads to a somewhat simplified derivation, which eventually yields the same amplitude equation to describe the slow dynamics of the system of resonators. We perform the same scaling of the equation parameters as we did in Sec. 11.3, with one difference-in anticipation of treating extremely large arrays, with thousands or more normal modes of vibration, we do not wish to assume that the width of the frequency band is small. We therefore do not replace $D$ with $\epsilon d$ as before. This will also allow us to obtain the exact dispersion relation (11.20 at the linear step, i.e. at order $\sqrt{\epsilon}$. Our starting point is therefore the set of coupled equations

$$
\ddot{u}_{n}+\epsilon \dot{u}_{n}+\left(1-\epsilon h \cos 2 \omega_{p} t\right) u_{n}+\frac{1}{2} D\left(u_{n+1}-2 u_{n}+u_{n-1}\right)+u_{n}^{3}+\eta u_{n}^{2} \dot{u}_{n}=0,
$$

where we have taken a negative sign for the parametric driving term to be consistent with the sign used by BCL, thus merely shifting the phase of the drive by $\pi$ relative to eqn (11.4).

Amplitude Equations for Counter Propagating Waves. In order to treat this system of equations analytically, beyond the treatment described earlier in Sec. 11.3, we introduce a continuous displacement field $u(x, t)$, keeping in mind that only for integral values $x=n$ of the spatial coordinate does it actually correspond to the displacements $u(n, t)=u_{n}(t)$ of the discrete set of resonators in the array. We introduce slow spatial and temporal scales, $X=\epsilon x$ and $T=\epsilon t$, upon which the dynamics of the envelope function occurs, and expand the displacement field in terms of $\epsilon$,

$$
\begin{aligned}
u(x, t) & =\epsilon^{1 / 2}\left[\left(A_{+}(X, T) e^{-i q_{p} x}+A_{-}^{*}(X, T) e^{i q_{p} x}\right) e^{i \omega_{p} t}+\text { c.c. }\right] \\
& +\epsilon^{3 / 2} u^{(1)}(x, t, X, T)+\ldots,
\end{aligned}
$$

where the asterisk and c.c. stand for the complex conjugate, and $q_{p}$ and $\omega_{p}$ are related through the dispersion relation,

$$
\omega_{p}^{2}=1-2 D \sin ^{2} \frac{q_{p}}{2} .
$$


Note that the response to lowest order in $\epsilon$ is expressed in terms of two counterpropagating waves with complex amplitudes $A_{+}$and $A_{-}$, which is a typical ansatz for parametrically excited continuous systems (Cross and Hohenberg, 1993). We substitute the ansatz 11.36 into the equations of motion (11.35) term by term. Again, using eqn 11.12 in addition to expanding $A_{ \pm}(X+\epsilon, T) \simeq A_{ \pm}(X, T)+\epsilon \partial A_{ \pm}(X, T) / \partial X$ we obtain up to order $\epsilon^{3 / 2}$,

$$
\begin{aligned}
\ddot{u}_{n} & =\epsilon^{1 / 2}\left[\left(-\omega_{p}^{2} A_{+}+2 i \omega_{p} \epsilon \frac{\partial A_{+}}{\partial T}\right) e^{-i q_{p} x}+\left(-\omega_{p}^{2} A_{-}^{*}+2 i \omega_{p} \epsilon \frac{\partial A_{-}^{*}}{\partial T}\right) e^{i q_{p} x}\right] e^{i \omega_{p} t} \\
& + \text { c.c. }+\epsilon^{3 / 2} \frac{\partial^{2} u^{(1)}}{\partial t^{2}} \\
u_{n \pm 1} & =\epsilon^{1 / 2}\left[\left(A_{+} \pm \epsilon \frac{\partial A_{+}}{\partial X}\right) e^{-i q_{p}(x \pm 1)}+\left(A_{-}^{*} \pm \epsilon \frac{\partial A_{-}^{*}}{\partial X}\right) e^{i q_{p}(x \pm 1)}\right] e^{i \omega_{p} t}+c . c . \\
& +\epsilon^{3 / 2} u^{(1)}(x \pm 1, t, X, T), \\
\frac{1}{2} D & \left(u_{n+1}-2 u_{n}+u_{n-1}\right)=-\epsilon^{1 / 2} 2 D \sin ^{2}\left(q_{p} / 2\right)\left(A_{+} e^{-i q_{p} x}+A_{-}^{*} e^{i q_{p} x}\right) e^{i \omega_{p} t} \\
& -\epsilon^{3 / 2} i D \sin \left(q_{p}\right)\left(\frac{\partial A_{+}}{\partial X} e^{-i q_{p} x}-\frac{\partial A_{-}^{*}}{\partial X} e^{i q_{p} x}\right) e^{i \omega_{p} t}+c . c . \\
& +\epsilon^{3 / 2} \frac{D}{2}\left[u^{(1)}(x+1, t, X, T)-2 u^{(1)}(x, t, X, T)+u^{(1)}(x-1, t, X, T)\right] \\
\epsilon h & \cos \left(2 \omega_{p} t\right) u_{n}=\epsilon^{3 / 2} \frac{h}{2}\left(A_{-} e^{-i q_{p} x}+A_{+}^{*} e^{i q_{p} x}\right) e^{i \omega_{p} t}+O\left(e^{i 3 \omega_{p} t}\right)+c . c ., \\
\epsilon \dot{u}_{n} & =\epsilon^{3 / 2} i \omega_{p}\left(A_{+} e^{-i q_{p} x}+A_{-}^{*} e^{i q_{p} x}\right) e^{i \omega_{p} t}+c . c ., \\
u_{n}^{3} & =\epsilon^{3 / 2} 3\left[\left(\left|A_{+}\right|^{2}+2\left|A_{-}\right|^{2}\right) A_{+} e^{-i q_{p} x}+\left(2\left|A_{+}\right|^{2}+\left|A_{-}\right|^{2}\right) A_{-}^{*} e^{i q_{p} x}\right] e^{i \omega_{p} t} \\
& +O\left(e^{i 3 \omega_{p} t}, e^{i 3 q_{p} x}\right)+c . c .,
\end{aligned}
$$

and

$$
\begin{aligned}
u_{n}^{2} \dot{u}_{n} & =\epsilon^{3 / 2} i \omega_{p}\left[\left(\left|A_{+}\right|^{2}+2\left|A_{-}\right|^{2}\right) A_{+} e^{-i q_{p} x}+\left(2\left|A_{+}\right|^{2}+\left|A_{-}\right|^{2}\right) A_{-}^{*} e^{i q_{p} x}\right] e^{i \omega_{p} t} \\
& +O\left(e^{i 3 \omega_{p} t}, e^{i 3 q_{p} x}\right)+c . c .
\end{aligned}
$$

where $O\left(e^{i 3 \omega_{p} t}, e^{i 3 q_{p} x}\right)$ are fast oscillating terms proportional to $e^{i 3 \omega_{p} t}$ or $e^{i 3 q_{p} x}$ that do not enter the dynamics at the lowest order in $\epsilon$ because they are nonsecular.

At the order of $\epsilon^{1 / 2}$, the equations of motion 11.35 are satisfied trivially, yielding the dispersion relation 11.37 mentioned earlier. At the order of $\epsilon^{3 / 2}$ on the other hand, we again obtain secular terms, and must apply a solvability condition, which requires that all terms proportional to $e^{i\left(\omega_{p} t \pm q_{p} x\right)}$ must vanish. As a result, we obtain the two coupled amplitude equations,

$$
\frac{\partial A_{ \pm}}{\partial T} \pm v_{g} \frac{\partial A_{ \pm}}{\partial X}=-\frac{1}{2} A_{ \pm} \mp \frac{i h}{4 \omega_{p}} A_{\mp}-\frac{1}{2}\left(\eta \mp \frac{3 i}{\omega_{p}}\right)\left(\left|A_{ \pm}\right|^{2}+2\left|A_{\mp}\right|^{2}\right) A_{ \pm},
$$

where the upper signs (lower signs) give the equation for $A_{+}\left(A_{-}\right)$, from the restriction on the terms proportional to $e^{i \omega_{p} t-i q_{p} x}\left(e^{i \omega_{p} t+i q_{p} x}\right)$, and where 


$$
v_{g}=\frac{\partial \omega}{\partial q}=-\frac{D \sin \left(q_{p}\right)}{2 \omega_{p}}
$$

is the group velocity. A detailed derivation of the amplitude equations $(11.39)$ can be found in the Masters thesis of Bromberg (2004). Similar equations were previously derived for describing Faraday waves (Ezerskil et al., 1986; Milner, 1991).

Reduction to a Single Amplitude Equation. By linearizing eqns (11.39) about the zero solution $\left(A_{+}=A_{-}=0\right)$ we find that the linear combination of the two amplitudes that first becomes unstable at $h_{c}=2 \omega_{p}$ is $\hat{B} \propto\left(A_{+}-i A_{-}\right)$-representing the emergence of a standing wave with a temporal phase of $\pi / 4$ relative to the drive - while the orthogonal linear combination of the amplitudes decays exponentially and does not participate in the dynamics at onset. Thus, just above threshold we can reduce the description of the dynamics to a single amplitude $\hat{B}$, where at a finite distance above threshold a band of unstable modes around $q_{p}$ can contribute to the spatial form of $\hat{B}$. This is similar to the procedure introduced by Riecke (1990) for describing the onset of Faraday waves.

To proceed with our multiple scales analysis, and obtain an equation describing the relevant slow dynamics of the new amplitude $\hat{B}$, we need to identify another physically small parameter with which we can associate even slower spatial and temporal scales. We therefore assume that the coefficient of nonlinear damping $\eta$ is small, and define a second small parameter $\delta=\eta^{2} \ll 1$. We then define a reduced driving amplitude $\hat{g}$ with respect to the threshold $h_{c}$ by letting $\left(h-h_{c}\right) / h_{c} \equiv \hat{g} \delta$. A sequence of judicious arguments (Bromberg, 2004, Bromberg et al., 2006) then encourages us to scale the original amplitudes $A_{ \pm}$as $\delta^{1 / 4}$, making the ansatz that

$$
\left(\begin{array}{c}
A_{+} \\
A_{-}
\end{array}\right)=\delta^{1 / 4}\left(\begin{array}{c}
1 \\
i
\end{array}\right) \hat{B}(\hat{\xi}, \hat{\tau})+\delta^{3 / 4}\left(\begin{array}{c}
w^{(1)}(X, T, \hat{\xi}, \hat{\tau}) \\
v^{(1)}(X, T, \hat{\xi}, \hat{\tau})
\end{array}\right)+\delta^{5 / 4}\left(\begin{array}{c}
w^{(2)}(X, T, \hat{\xi}, \hat{\tau}) \\
v^{(2)}(X, T, \hat{\xi}, \hat{\tau})
\end{array}\right)+\ldots
$$

where $\hat{\xi}=\delta^{1 / 2} X$ and $\hat{\tau}=\delta T$ are the new spatial and temporal scales respectively.

We substitute the ansatz (11.41) into the coupled amplitude equations (11.39) and collect terms of different orders in $\delta$. Again, to the lowest order of expansion the equations are satisfied trivially. Collecting all terms of order $\delta^{3 / 4}$ in eqns 11.39 yields

$$
\mathfrak{O}\left(\begin{array}{c}
w^{(1)} \\
v^{(1)}
\end{array}\right)=\left(-v_{g} \frac{\partial \hat{B}}{\partial \hat{\xi}}+i \frac{9}{2 \omega_{p}}|\hat{B}|^{2} \hat{B}\right)\left(\begin{array}{c}
1 \\
-i
\end{array}\right),
$$

where exactly at onset $\mathfrak{O}$ is a linear operator given by the matrix

$$
\left(\begin{array}{cc}
\partial_{T}+v_{g} \partial_{X}+\frac{1}{2} & \frac{i}{2} \\
-\frac{i}{2} & \partial_{T}-v_{g} \partial_{X}+\frac{1}{2}
\end{array}\right)
$$

The vector $\left(\begin{array}{c}1 \\ -i\end{array}\right)$, on the right hand side of eqn 11.42 is an eigenvector of $\mathfrak{O}$, with an eigenvalue -1 . The solution of eqn 11.42 is therefore immediately given by

$$
\left(\begin{array}{c}
w^{(1)} \\
v^{(1)}
\end{array}\right)=\left(-v_{g} \frac{\partial \hat{B}}{\partial \hat{\xi}}+i \frac{9}{2 \omega_{p}}|\hat{B}|^{2} \hat{B}\right)\left(\begin{array}{c}
1 \\
-i
\end{array}\right) .
$$


We substitute eqn 11.44 back into eqns 11.39 , collect all the terms of order $\delta^{5 / 4}$ and obtain

$$
\begin{aligned}
\mathfrak{O}\left(\begin{array}{c}
w^{(2)} \\
v^{(2)}
\end{array}\right)= & {\left[-\frac{\partial \hat{B}}{\partial \hat{\tau}}+v_{g}^{2} \frac{\partial^{2} \hat{B}}{\partial \hat{\xi}^{2}}+\frac{\hat{g}}{2} \hat{B}-\frac{3}{2}|\hat{B}|^{2} \hat{B}\right.} \\
& \left.\quad-i \frac{3 v_{g}}{\omega_{p}}\left(4|\hat{B}|^{2} \frac{\partial \hat{B}}{\partial \hat{\xi}}+\hat{B}^{2} \frac{\partial \hat{B}^{*}}{\partial \hat{\xi}}\right)-\left(\frac{9}{2 \omega_{p}}\right)^{2}|\hat{B}|^{4} \hat{B}\right]\left(\begin{array}{l}
1 \\
i
\end{array}\right) .
\end{aligned}
$$

The vector $\left(\begin{array}{l}1 \\ i\end{array}\right)$, on the right hand side of eqn 11.45 , is an eigenvector of $\mathfrak{O}$ with zero eigenvalue. Clearly, the left hand side of the equation cannot contain any component along the direction of such a zero eigenvector. Therefore, the expression within the square brackets is a secular term that must vanish. This provides us with the required solvability condition to proceed (Cross and Hohenberg, 1993). After applying one last set of rescaling transformations,

$$
\hat{\tau}=\frac{36}{\omega_{p}^{2}} \tau, \quad \hat{\xi}=\frac{6\left|v_{g}\right|}{\omega_{p}} \xi, \quad \hat{B}=\frac{\omega_{p}}{3 \sqrt{3}} B, \quad \text { and } \quad \hat{g}=\frac{\omega_{p}^{2}}{18} g,
$$

we end up with the BCL amplitude equation, which is governed by a single parameter,

$$
\frac{\partial B}{\partial \tau}=g B+\frac{\partial^{2} B}{\partial \xi^{2}}+i \frac{2}{3}\left(4|B|^{2} \frac{\partial B}{\partial \xi}+B^{2} \frac{\partial B^{*}}{\partial \xi}\right)-2|B|^{2} B-|B|^{4} B .
$$

\subsubsection{Analyzing and solving the equation}

The simplest nontrivial solutions of the BCL amplitude equation (11.47) are steadystate single-mode extended patterns, given by

$$
B(\xi, \tau)=b_{k} e^{i(\varphi-k \xi)},
$$

with $b_{k}$ and $\varphi$ both real, and where the boundary conditions $u(0, t)=u(N+1, t)=$ 0 constrain the phase $\varphi$ to be $\pi / 4$ or $5 \pi / 4$. In steady state, the relation between the magnitude $b_{k}$ and the wave number $k$ is found by substituting eqn (11.48) into eqn (11.47), and setting the time derivative to zero to give

$$
b_{k}^{2}=(k-1)+\sqrt{(k-1)^{2}+\left(g-k^{2}\right)} \geq 0,
$$

along with a negative square-root branch which is always unstable against small perturbations, as can be verified by the analysis below.

Substituting the single-mode solution of eqn (11.48), with $\varphi=\pi / 4$, back into eqn (11.44) and eqn (11.41), and then into eqn (11.36), yields extended single-mode standing-wave parametric oscillations at half the drive frequency, whose explicit form is given by

$$
u(x, t) \simeq \epsilon^{1 / 2} \delta^{1 / 4} \frac{4 \omega_{p} \sqrt{1+\tan ^{2}(\alpha)}}{3 \sqrt{3}} b_{k} \sin \left(q_{m} x\right) \cos \left(\pi / 4-\omega_{p} t-\alpha\right),
$$


where we have defined

$$
\tan (\alpha) \equiv \delta^{1 / 2} \frac{\omega_{p}}{6}\left(b_{k}^{2}-k\right) .
$$

To satisfy the boundary conditions $u(0, t)=u(N+1, t)=0$, the wave numbers $q_{m}$ must be of the form

$$
q_{m}=\frac{m \pi}{N+1}=q_{p}+\frac{k \pi}{\Delta Q_{N}(N+1)},
$$

where

$$
\Delta Q_{N}=\frac{1}{\epsilon \delta^{1 / 2}} \frac{3 D \sin \left(q_{p}\right)}{\omega_{p}^{2}} \frac{\pi}{N+1} .
$$

BCL showed that the first single-mode pattern to emerge as the zero-state becomes unstable is the one whose wave number $q_{m}$ is closest to the wave number $q_{p}$ that is determined by the drive frequency $\omega_{p}$ through the dispersion relation (11.37). This determines the value of the scaled wave number in the single-mode solution (11.48) to be

$$
k_{0}=\left(m-q_{p} \frac{N+1}{\pi}\right) \Delta Q_{N},
$$

where $m$ is the integer closest to $q_{p}(N+1) / \pi$. Note that $\Delta Q_{N}$ tends to zero as the size $N$ of the array of resonators tends to infinity.

Linearization of the BCL amplitude equation (11.47) shows that the zero state with $B(\xi, \tau)=0$ - which is a solution of eqn (11.47) for any value of $g$ - is stable against the formation of single-mode patterns with wave number $k$ as long as $g<k^{2}$. The neutral stability curve $g=k^{2}$ is plotted as a dashed parabola in Fig. 11.3. Furthermore, for $k<1$ the bifurcation from the zero state to that of single-mode oscillations is supercritical, occurring on the neutral stability curve, while for $k>1$ it is subcritical, with a locus of saddle-node bifurcations located along the line $g=2 k-1$ (shown in Fig. 11.3 as a solid green line), where the square root in eqn $(11.49$ is exactly zero.

The stability of a single-mode solution (11.48) of wave number $k$ against an Eckhaus transition to a different single-mode solution of wave number $k \pm Q$ is found by performing a linear stability analysis of solutions of the form

$$
B(\xi, \tau)=b_{k} e^{-i k \xi}+\left(\beta_{+}(\tau) e^{-i(k+Q) \xi}+\beta_{-}^{*}(\tau) e^{-i(k-Q) \xi}\right),
$$

with $\left|\beta_{ \pm}\right| \ll 1$. When the larger of the two eigenvalues describing the growth of such a perturbation becomes positive the single-mode solution of wave number $k$ undergoes an Eckhaus instability with respect to single-mode solutions of wave numbers $k \pm Q$.

For an infinite number of oscillators the Eckhaus instability forms the upper boundary of the stability balloon of the single-mode solutions, and also the lower boundary for $k<5 / 2$. For $k>5 / 2$ the lower boundary is the saddle node bifurcation line. For a finite number of oscillators, restricting $Q$ to be an integer multiple of $\Delta Q_{N}$ in eqn 11.55 slightly shifts the Eckhaus instability lines. The upper Eckhaus boundary is shifted to larger values of $g$. The nature of the lower instability boundary now depends on the number of resonators in the array through $\Delta Q_{N}$, as well as on the wave number $k$. For $k<1$ the lower boundary will be the Eckhaus instability curve if $|k|>\Delta Q_{N} / 2$, and the neutral stability curve otherwise. Because the only wave 
number to satisfy $|k|<\Delta Q_{N} / 2$ is $k_{0}$, given by eqn 11.54, upon decreasing $g$ the $k_{0}$ solution undergoes a continuous transition to the zero state. For $k>1$ the lower boundary will be the Eckhaus instability curve if $1<k<\left(5-3\left(\Delta Q_{N} / 2\right)^{2}\right) / 2$, and the line of saddle node bifurcations otherwise. For $\Delta Q_{N}>2$ there is no portion of Eckhaus instability on the lower boundary, which is the neutral stability curve if $k<1$ and the saddle node bifurcation curve if $k>1$. These stability boundaries are shown in Fig. 11.3 for an infinite system and for a system of $N=92$ resonators. Further details can be found in Bromberg et al. (2006) and Kenig et al. (2009a).

\subsubsection{Brief survey of applications}

Kenig et al. (2009a) used the BCL amplitude equation (11.47) to study a number of collective dynamical effects in one-dimensional arrays of coupled nonlinear resonators. The common thread linking these effects is the so-called question of pattern selection

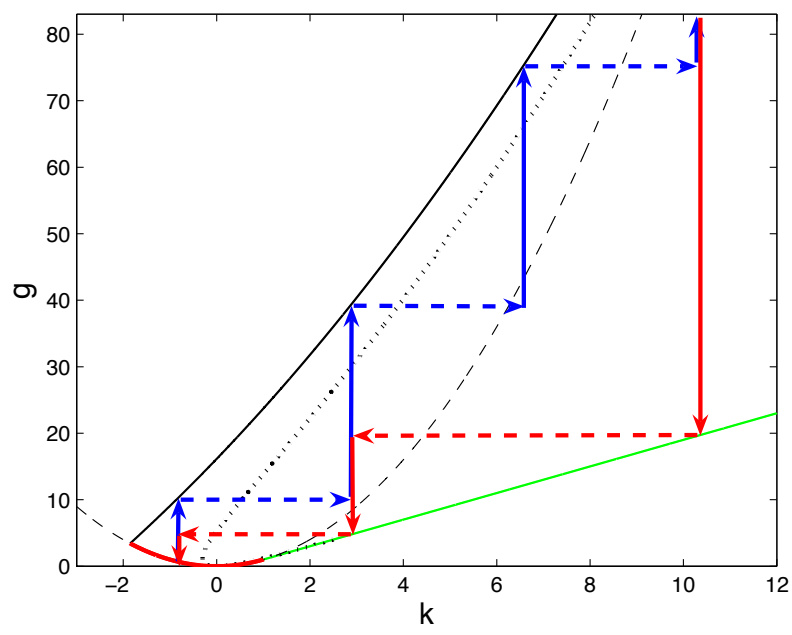

Fig. 11.3 (Color) Stability boundaries of the single-mode solution 11.48 of the BCL amplitude equation 11.47 in the $g$ vs. $k$ plane. Dashed line: neutral stability curve $g=k^{2}$. Dotted line: stability boundary of the single-mode solution 11.48 for a continuous spectrum $\left(\Delta Q_{N} \rightarrow 0\right)$. Solid lines: stability boundary of the single-mode solution for $N=92$ and the parameters $D=0.25, q_{p}=73 \pi / 101$, and $\epsilon=\delta=0.01$ (giving $k_{0} \simeq-0.81$ and $\Delta Q_{N} \simeq 3.70$ ). Black line: the value of $g$ for which perturbations of the form given by eqn 11.55 start to grow. Red line: the lower bound for $k<1, g=k^{2}$. Green line: the lower bound for $k>1$, the locus of saddle-node bifurcations $g=2 k-1$. Vertical and horizontal arrows mark the secondary instability transitions that are expected upon quasistatic sweeps of $g$. The blue upward-pointing arrows are for upward sweeps that undergo an Eckhaus instability, and the red downward-pointing arrows are for downward sweeps, of which the two with $k>1$ experience a saddle-node bifurcation, and the one with $k<1$ goes through a continuous (supercritical) bifurcation. From Kenig et al. (2009a). Copyright (2009) American Physical Society. 


\section{Collective Dynamics in Arrays of Coupled Nonlinear Resonators}

(Cross and Greenside, 2009) — the nonlinear competition between different single-mode standing-wave patterns of the form of eqn 11.50, when many such solutions are simultaneously stable. This question becomes particularly interesting when the control parameter - in our case the drive amplitude $g$ - is varied as a function of time, either quasistatically, abruptly, or in an intermediate ramp rate. In all such cases one is interested in predicting which of all stable patterns will be selected, as well as in the detailed understanding of the nature of the switching between patterns as their stability changes.

The BCL amplitude equation allowed Kenig et al. (2009a) to map out the expected behavior of the resonators using universal stability diagrams, like the one shown in Fig. 11.3. Such a diagram immediately shows the type of instability that will be encountered upon variation of the control parameter, and gives qualitative insights on the mode jumps to be expected. For example, for quasistatic parameter variations the jump in the mode number is always unity if the control parameter is increased so that the Eckhaus instability operates, but larger jumps are often seen if the control parameter is decreased so that a saddle-node bifurcation occurs. This is indicated by the dashed sideways arrows in Fig. 11.3 .

It is instructive to describe how Kenig et al. (2009a) examined the process by which these two types of pattern switchings occur. To do so, we expand the general solution of the BCL amplitude equation in the linear modes of the array

$$
B(\xi, \tau)=\sum_{n} b_{n}(\tau) e^{i\left(\varphi_{n}-k_{n} \xi\right)},
$$

where $k_{n} \equiv k_{0}+n \Delta Q_{N}$, and $k_{0}$ is defined in eqn 11.54). Substituting a truncated mode expansion $(11.56)$ containing a finite number of modes around $k_{0}$ into the BCL amplitude equation (11.47), allows us to replace this partial differential equation with a finite number of ordinary differential equations for the coupled mode amplitudes,

$$
\begin{aligned}
\frac{\partial b_{n}}{\partial \tau} & =\left(g-k_{n}^{2}\right) b_{n}+2 \sum_{m, p}\left(k_{p}-1-\frac{m-n}{3} \Delta Q_{N}\right) b_{m} b_{p} b_{m+p-n}^{*} \\
& -\sum_{m, l, p, r} b_{m} b_{l}^{*} b_{p} b_{r} b_{m-l+p+r-n}^{*} .
\end{aligned}
$$

To satisfy the boundary conditions, as mentioned above for the single-mode solution 11.48, we take each mode amplitude to be zero at the boundaries by setting all the phases $\varphi_{n}$ in Eq. (11.56) to $\pi / 4$, and take the amplitudes $b_{n}$ to be real, keeping in mind that they can be either positive or negative. Note that if all mode amplitudes except $b_{0}$ are set to zero we obtain a single equation with $n=m=p=l=r=0$, whose steady-state solution is the same as the single-mode solution of BCL $(11.49$ ).

We take a closer look at the transient behavior during the first Eckhaus transition from the initial $k_{0}$ pattern to the $k_{1}$ pattern by plotting the time evolution of the four largest modes, as shown in Fig. 11.4(a). One can observe the decay of the unstable mode amplitude $b_{0}$ followed by the growth of $b_{1}$ to its steady-state value. One can also see that during the transient the amplitude of the unstable mode $b_{-1}$ becomes nonzero. Its participation in the Eckhaus transition from the $k_{0}$ pattern to the $k_{1}$ pattern 
is essential, as can be verified by considering these two modes alone in a truncated expansion. Limiting the expansion to $b_{0}$ and $b_{1}$ suppresses the Eckhaus transition, and the $k_{0}$ pattern remains stable as $g$ exceeds its expected value for the Eckhaus instability. The Eckhaus transition is observed only when the $k_{-1}$ mode is included as well, corresponding to the stability calculation, performed earlier for the state given by Eq. 11.55).

One might naively expect that the same mechanism causes the transition from the $k_{3}$ pattern to the $k_{1}$ pattern at $g=19$ through a double phase slip (with $Q=$ $2 \Delta Q_{N}$ ), however, this is not the case. Fig. 11.4(b) reveals the transient processes on a downward sweep of $g$ just below the saddle node at $g=19$. As $g$ crosses the saddlenode bifurcation point, the amplitude $b_{3}$ drops abruptly to zero. As can be seen from Eq. (11.57), in the zero-displacement state the linear growth rates of the solutions 11.56) are $g-k_{n}^{2}$, so the $k_{0}$ pattern has the largest possible growth rate and it outgrows the other modes until its amplitude approaches the steady state value (11.49). However, at this value of $g$ the $k_{0}$ pattern is Eckhaus unstable with respect to the $k_{1}$ pattern - notice the characteristic evolution of the modes around $\tau=3$ in Fig. 11.4(b) corresponding to the Eckhaus instability [cf. around $\tau=25$ in Fig. 11.4(a)]. Thus the $k_{1}$ mode is ultimately the selected pattern.

For more rapid increases in the control parameter larger jumps in the mode number may occur, and these were shown to be predicted simply from a linear stability analysis following the Eckhaus instability. Kenig et al. (2009a) showed that following an abrupt

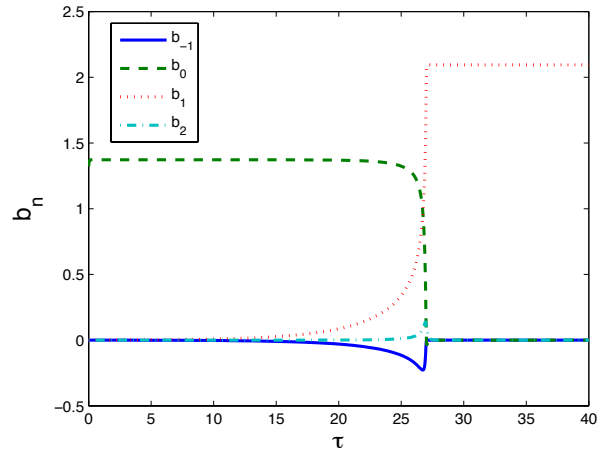

(a) Eckhaus instability

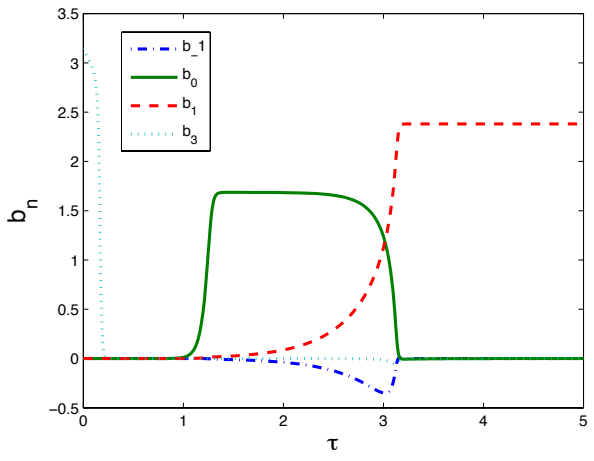

(b) Saddle-node bifurcation

Fig. 11.4 Time evolution of the amplitudes of the four largest modes that participate in (a) the transition from the initial $k_{0}$ pattern to the $k_{1}$ pattern, when the value of the control parameter is changed from $g=10$ to $g=11$, causing the initial $k_{0}$ pattern to experience an Eckhaus instability; and (b) the transition from the $k_{3}$ pattern to the $k_{1}$ pattern, when the value of the control parameter is changed from $g=20$ to $g=19$, causing the $k_{3}$ pattern to go through a saddle-node bifurcation. In both cases the results are obtained by a numerical integration of the seven truncated mode equations (11.57), for modes $b_{-3}$ to $b_{3}$, using the same parameters as in Fig. 11.3 Details of the transitions are discussed in the text. From Kenig et al. (2009a). Copyright (2009) American Physical Society. 
increase of $g$ that crosses the Eckhaus instability line it is simply the mode whose linear growth rate is greatest that is selected. For a slow temporal ramp of the control parameter $g=\alpha \tau$, with $\alpha \ll 1$, they encountered a more interesting competition between the different patterns. They showed that as the control parameter is ramped a sequence of patterns start to grow one by one, yet the growth rates increase with each pattern that emerges. This resembles a balanced race in which the slow runners are allowed to start running before the fast ones. Nevertheless, Kenig et al. (2009a) were able to predict the winning pattern, and its dependence on the ramp rate $\alpha$. In all cases that were checked, simulations of the original equations of motion of the resonators (11.35) confirm the results based on the BCL amplitude equation.

\subsection{Continuous Amplitude Equations: Example IV - Intrinsic localized modes (ILMs)}

\subsubsection{Derivation of the PDNLS equation}

As our final example we focus on a different type of nonlinear states, namely, intrinsic localized modes (ILMs), also known as discrete breathers or lattice solitons (Ovchinnikov and Érikhman, 1982, Sievers and Takeno, 1988, Campbell et al., 2004 Maniadis and Flach, 2006). These localized states are intrinsic in the sense that they arise from the inherent nonlinearity of the resonators, rather than from extrinsicallyimposed disorder as in the case of Anderson localization. ILMs were observed by Sato et al. $(2003 a, 2003 b, 2004,2006,2007,2008)$ in driven arrays of micromechanical resonators. They were also observed in a wide range of other physical systems including coupled arrays of Josephson junctions (Trías et al., 2000; Binder et al., 2000), coupled optical waveguides (Eisenberg et al., 1998; Eisenberg et al., 2001: Cheskis et al., 2003), two-dimensional nonlinear photonic crystals (Fleischer et al., 2003), highly-nonlinear atomic lattices (Swanson et al., 1999), and antiferromagnets (Schwarz et al., 1999: Sato and Sievers, 2004). Thus, the ability to perform a quantitative comparison between our theory and future experiments with large arrays of MEMS and NEMS resonators, may have consequences far beyond the framework of mechanical systems considered here.

We follow the work of Kenig et al. (2009b), whose goal was to predict the actual physical parameters, in realistic arrays of MEMS and NEMS resonators, for which ILMs can form and sustain themselves. Such predictions may have practical consequences for actual applications exploiting self-localization to focus energy, and others that may want to avoid energy focusing, for example in cases where very large oscillation amplitudes may lead to mechanical failure. Again we wish to formulate our analysis in terms of a continuous amplitude equation and to display the range of stable ILMs on a reduced diagram - as we did for extended modes in Fig. 11.3 -helping to describe the general qualitative behavior as physical parameters are varied.

We start with the same form of the equations of motion 111.35 that we used in the preceding section, with two differences: (1) We wish to keep an explicit parameter with which we can vary the linear damping, thus we define the small expansion parameter as $Q^{-1}=\epsilon \hat{\gamma}$, with $\epsilon \ll 1$, and $\hat{\gamma}$ of order unity; and (2) We use a negative sign before the coupling coefficient $D$ to model elastic coupling between adjacent beams, which 
is stronger as the separation between neighbors increases, thus acting to stiffen the resonators. This leads to a dispersion curve that has a positive slope, or a positive group velocity. The coupling mechanism in the experimental setups in which ILMs were observed by Sato et al. (2003a, 2003b, 2004, 2006, 2007, 2008) is of this kind. Our equations of motion then become

$$
\ddot{u}_{n}+\epsilon \hat{\gamma} \dot{u}_{n}+\left(1-\epsilon \hat{h} \cos 2 \omega_{p} t\right) u_{n}-\frac{1}{2} D\left(u_{n+1}-2 u_{n}+u_{n-1}\right)+u_{n}^{3}+\hat{\eta} u_{n}^{2} \dot{u}_{n}=0
$$

with hats to be removed later by additional scaling.

An experimental protocol for producing ILMs in an array of resonators with a stiffening nonlinearity - albeit not the one we use below - is to drive the array at the highest-frequency extended mode. As the resonators are collectively oscillating at this mode, the frequency is raised further, which through the stiffening Duffing nonlinearity results in an increase of the oscillation amplitude up to a point at which the extended pattern breaks into localized modes (Sato et al., 2003a: Sato et al., 2006). With this in mind - and concentrating on the case of elastic coupling where the highest-frequency mode $\omega=\sqrt{1+2 D}$ is the staggered mode, in which adjacent resonators oscillate out of phase - we write the displacement of the $n^{\text {th }}$ resonator as

$$
u_{n}=\epsilon^{1 / 2}\left[\hat{\psi}\left(\hat{X}_{n}, \hat{T}\right) e^{i(\omega t-\pi n)}+c . c .\right]+\epsilon^{3 / 2} u_{n}^{(1)}\left(t, \hat{T}, \hat{X}_{n}\right)+\ldots
$$

with slow temporal and spatial variables $\hat{T}=\epsilon t$ and $\hat{X}_{n}=\epsilon^{1 / 2} n$. As usual, we take the parametric drive frequency to be close to twice $\omega$ by setting $\omega_{p}=\omega+\epsilon \Omega / 2$, introduce a continuous spatial variable $\hat{X}$ in place of $\hat{X}_{n}$, and substitute the ansatz 11.59 into the equations of motion 11.58 term by term. Up to order $\epsilon^{3 / 2}$ we have

$$
\begin{aligned}
& \ddot{u}_{n}=\epsilon^{1 / 2}\left[\left(-\omega^{2} \hat{\psi}+2 i \omega \epsilon \frac{\partial \hat{\psi}}{\partial \hat{T}}\right) e^{i(\omega t-\pi n)}+c . c .\right]+\epsilon^{3 / 2} \ddot{u}_{n}^{(1)}, \\
& u_{n \pm 1}=-\epsilon^{1 / 2}\left[\left(\hat{\psi} \pm \epsilon^{1 / 2} \frac{\partial \hat{\psi}}{\partial \hat{X}}+\frac{\epsilon}{2} \frac{\partial^{2} \hat{\psi}}{\partial \hat{X}^{2}}\right) e^{i(\omega t-\pi n)}+c . c .\right]+\epsilon^{3 / 2} u_{n \pm 1}^{(1)}, \\
& \epsilon \hat{h} \cos \left(2 \omega_{p} t\right) u_{n}=\epsilon^{3 / 2} \frac{\hat{h}}{2} \hat{\psi}^{*} e^{i \Omega \hat{T}} e^{i(\omega t+\pi n)}+O\left(e^{i 3 \omega t}\right)+c . c . \\
& \epsilon \hat{\gamma} \dot{u}_{n}=\epsilon^{3 / 2} \hat{\gamma} i \omega \hat{\psi} e^{i(\omega t-\pi n)}+c . c . \\
& u_{n}^{3}=\epsilon^{3 / 2} 3|\hat{\psi}|^{2} \hat{\psi} e^{i(\omega t-\pi n)}+O\left(e^{i 3 \omega t}, e^{i 3 \pi n}\right)+c . c .
\end{aligned}
$$

and

$$
u_{n}^{2} \dot{u}_{n}=\epsilon^{3 / 2} i \omega|\hat{\psi}|^{2} \hat{\psi} e^{i(\omega t-\pi n)}+O\left(e^{i 3 \omega t}, e^{i 3 \pi n}\right)+c . c .
$$

where $O\left(e^{i 3 \omega t}, e^{i 3 \pi n}\right)$ are fast oscillating terms with temporal frequency $3 \omega$ or spatial wavenumber $3 \pi$.

At order $\epsilon^{1 / 2}$ the equations of motion 11.58 are satisfied trivially. However, once again at order $\epsilon^{3 / 2}$ we encounter secular terms - in this case, proportional to $e^{i(\omega t-\pi n)}$ - and must apply a solvability condition, requiring all such terms to vanish. 


\section{Collective Dynamics in Arrays of Coupled Nonlinear Resonators}

Again, it is this condition that leads to a partial differential equation (PDE) describing the slow dynamics of the amplitudes of the resonators,

$$
2 i \omega \frac{\partial \hat{\psi}}{\partial \hat{T}}+(3+i \omega \hat{\eta})|\hat{\psi}|^{2} \hat{\psi}+\frac{1}{2} D \frac{\partial^{2} \hat{\psi}}{\partial \hat{X}^{2}}+i \hat{\gamma} \omega \hat{\psi}-\frac{\hat{h}}{2} \hat{\psi}^{*} e^{i \Omega \hat{T}}=0 .
$$

Note that while $e^{i(\omega t+\pi n)}=e^{i(\omega t-\pi n)}$, if we were to consider an arbitrary mode of wave number $q$ instead of $\pi$, the parametric term would have forced us to apply another solvability condition, requiring terms proportional to $e^{i(\omega t+q n)}$ to vanish. This was exactly the situation in the preceding section 11.5, where we were forced first to consider an ansatz based on counter propagating waves as the $O\left(\epsilon^{1 / 2}\right)$ solution for $u_{n}$, leading to a system of two coupled amplitude equations 11.39 , after which a second scaling was used to obtain a single amplitude equation 11.47). Here we can get away with a single step.

By means of rescaling,

$$
\hat{\psi}=\sqrt{\frac{2 \omega \Omega}{3}} \psi, \quad \hat{X}=\sqrt{\frac{D}{2 \omega \Omega}} X, \quad \hat{T}=\frac{2}{\Omega} T, \quad \hat{h}=2 \omega \Omega h, \quad \hat{\gamma}=\Omega \gamma, \quad \hat{\eta}=\frac{3}{2 \omega} \eta,
$$

we transform eqn 11.61 into a normalized form,

$$
i \frac{\partial \psi}{\partial T}=-\frac{\partial^{2} \psi}{\partial X^{2}}-i \gamma \psi-(2+i \eta)|\psi|^{2} \psi+h \psi^{*} e^{2 i T}
$$

We then perform one final transformation $\psi \rightarrow \psi e^{i T}$ and arrive at an autonomous $\mathrm{PDE}$, which is the final form of our amplitude equation,

$$
i \frac{\partial \psi}{\partial T}=-\frac{\partial^{2} \psi}{\partial X^{2}}+(1-i \gamma) \psi-(2+i \eta)|\psi|^{2} \psi+h \psi^{*}
$$

Equation 11.63 with $\eta=0$ is called the parametrically driven damped nonlinear Schrödinger equation (PDNLS). It models parametrically driven media in hydrodynamics (Zhang and Viñals, 1995, Wang and Wei, 1997; Wang and Wei, 1998, Miao and Wei, 1999) and optics (Longhi, 1996; Sánchez-Morcillo et al., 2000), and was also used as an amplitude equation to study localized structures in arrays of coupled pendulums (Denardo et al., 1992, Chen, 1994, Alexeeva et al., 2000). Recently, a pair of linearly-coupled PDNLS equations was used to model coupled dual-core wave guides (Dror and Malomed, 2009). Equation (11.64) has the form of a forced complex Ginzburg-Landau equation (Burke et al., 2008) but with specific coefficients that are derived, via the scaling performed in (11.59) and (11.62), from the underlying equations of motion (11.58). We note that considering the equations of motion 11.3 (yet still with a negative sign before $D$ ) as our stating point instead of eqns (11.58) leads to the same eqn (11.61) as above, but with slightly different coefficients. Thus, applying modified scaling (11.62) yields exactly the same amplitude equation (11.64). 


\subsubsection{Analyzing and solving the equation}

A remarkable feature of the amplitude equation 111.64 is that for $\eta=0$ it has exact steady-state solitonic solutions, as shown by Barashenkov et al. (1991),

$$
\Psi_{ \pm}(X)=A_{ \pm} e^{-i \Theta \pm} \operatorname{sech}\left[A_{ \pm}\left(X-X_{0}\right)\right],
$$

where $X_{0}$ is an arbitrary position of the soliton,

$$
A_{ \pm}^{2}=1 \pm \sqrt{h^{2}-\gamma^{2}} \text {, and } \cos \left(2 \Theta_{ \pm}\right)= \pm \sqrt{1-\frac{\gamma^{2}}{h^{2}}} \text {. }
$$

This pair of solitonic solutions exists for $\gamma<h$, above the dotted line in Fig. 11.5. It was shown by Barashenkov et al. (1991) that the $\Psi_{-}$soliton is unstable for all values of $\gamma$ and $h$, while the $\Psi_{+}$soliton is stable in a certain parameter range. A simple linear stability analysis shows that the zero solution $\psi(X)=0$, which exists for all parameter values, is stable only for $h<\sqrt{1+\gamma^{2}}$. This inequality, indicated by a dashed line in Fig. 11.5. also determines an upper stability limit for localized solutions of eqn (11.64, as they decay exponentially to zero on either side.

We follow Kenig et al. (2009b) in constructing an approximate analytical expression for the localized solution of the full amplitude equation (11.64), with $\eta>0$, implementing a method introduced by Barashenkov et al. (2003). To this end, we consider a function of the same form as $\Psi_{ \pm}$

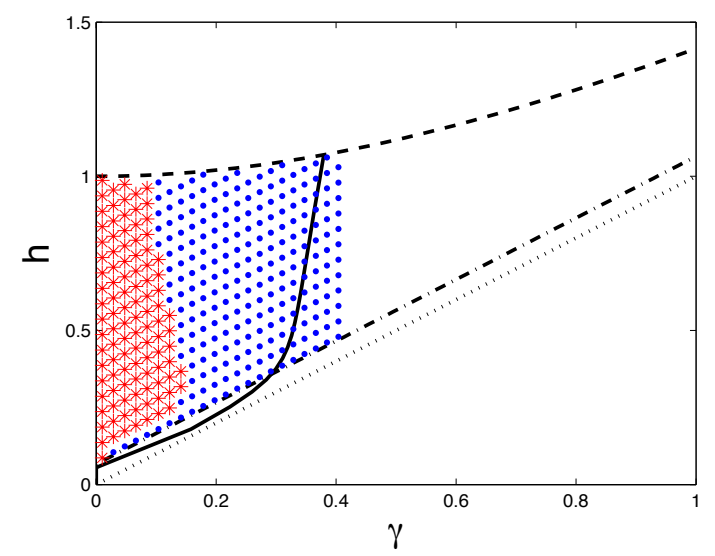

Fig. 11.5 Stability diagram for localized solutions of the amplitude equation 11.64 in the $h$ vs. $\gamma$ plane. The dotted line is the lower existence boundary for $\eta=0$, namely $h=\gamma$. The dash-dotted line is the approximate low boundary for $\eta=0.1$, given by eqn (11.74). Above the solid line the $\Psi_{+}$solution of the PDNLS equation with $\eta=0$ is unstable with respect to a Hopf bifurcation. The dashed line is the line $h=\sqrt{1+\gamma^{2}}$ above which the zero solution is unstable. Red $*$ s are points for which linear stability analysis shows that perturbations away from the soliton solution $\psi(X)$ grow exponentially for $\eta=0.1$, hence the soliton solution is unstable. Blue dots represent points for which the solution $\psi(X)$ is stable according to the linear analysis. From Kenig et al. (2009b). Copyright (2009) American Physical Society. 


$$
\psi(X, T)=a(T) e^{-i \theta(T)} \operatorname{sech}\left[a(T)\left(X-X_{0}\right)\right],
$$

except that $a$ and $\theta$ are now time-dependent. We multiply Eq. 11.64 by $\psi^{*}$, subtract the complex conjugate of the resulting equation and get

$$
i \frac{\partial|\psi|^{2}}{\partial T}=-\frac{\partial}{\partial X}\left(\frac{\partial \psi}{\partial X} \psi^{*}-\psi \frac{\partial \psi^{*}}{\partial X}\right)+h\left[\left(\psi^{*}\right)^{2}-\psi^{2}\right]-2 i \gamma|\psi|^{2}-2 i \eta|\psi|^{4} .
$$

By substituting $\psi=|\psi| e^{-i \chi}$, integrating over $X^{\prime}=X-X_{0}$, and assuming that $\psi \rightarrow 0$ and $\partial \psi / \partial X \rightarrow 0$ as $|X| \rightarrow \infty$, we obtain a spatially-independent integral equation

$$
\frac{d}{d T} \int|\psi|^{2} d X^{\prime}=2 \int|\psi|^{2}[h \sin (2 \chi)-\gamma] d X^{\prime}-2 \eta \int|\psi|^{4} d X^{\prime} .
$$

Substituting the ansatz (11.67) into eqn (11.69), we obtain the time evolution equation for $a$

$$
\frac{d a}{d T}=2 a\left(h \sin (2 \theta)-\gamma-\tilde{\eta} a^{2}\right),
$$

where $\tilde{\eta}=2 \eta / 3$. The time evolution equation for $\theta$ is derived in a similar way by multiplying eqn (11.64 by $\psi^{*}$, adding the complex conjugate of the resulting equation, substituting the ansatz (11.67), and integrating over space to yield

$$
\frac{d \theta}{d T}=h \cos (2 \theta)+1-a^{2} .
$$

Equations 11.70 and 11.71 have the same form as the equations obtained by Barashenkov et al. (2003), whose fixed points are

$$
a_{ \pm}^{2}=\frac{1-\gamma \tilde{\eta} \pm \sqrt{h^{2}\left(1+\tilde{\eta}^{2}\right)-(\gamma+\tilde{\eta})^{2}}}{1+\tilde{\eta}^{2}}
$$

which has to be positive, and

$$
\begin{aligned}
h \cos \left(2 \theta_{ \pm}\right) & =a_{ \pm}^{2}-1 \\
h \sin \left(2 \theta_{ \pm}\right) & =\gamma+\tilde{\eta} a_{ \pm}^{2} .
\end{aligned}
$$

A linear analysis of these stationary points shows that $\left(a_{+}, \theta_{+}\right)$and $\left(a_{-}, \theta_{-}\right)$are a stable node and a saddle, respectively (Barashenkov et al., 2003). The saddle-node bifurcation point of these solutions occurs at

$$
h_{s n}(\tilde{\eta})=\frac{\gamma+\tilde{\eta}}{\sqrt{1+\tilde{\eta}^{2}}}, \quad \text { where } \quad \tilde{\eta}=\frac{2}{3} \eta,
$$

as long as $\gamma \tilde{\eta}<1$. This is the approximate minimal driving strength required to support a localized structure in the array, in the presence of linear and nonlinear dissipation. It is indicated by a dash-dotted line in Fig 11.5 for $\eta=0.1$. 
The approximate stable localized solution of the amplitude equation 11.64 is therefore given by

$$
\psi_{\text {app }}(X)=a_{+} e^{-i \theta_{+}} \operatorname{sech}\left(a_{+}\left(X-X_{0}\right)\right) .
$$

Substituting this expression into eqn 11.59 yields an approximate expression for the displacements of the actual resonators in the array of the form

$$
u_{n}(t) \simeq 2 \sqrt{\frac{2 \epsilon \omega \Omega}{3}} a_{+} \operatorname{sech}\left[a_{+}\left(\sqrt{\frac{2 \epsilon \omega \Omega}{D}} n-X_{0}\right)\right] \cos \left(\omega_{p} t-\pi n-\theta_{+}\right) .
$$

To obtain accurate solutions one has no choice but to solve the amplitude equation, or the underlying discrete equations of motion, numerically. We do so with the equations of motion (11.58) by initiating them with the approximate expression (11.76) at a value of $h$ just above the saddle node $h_{s n}$ (11.74). We then perform a quasistatic upward sweep of $h$, raising $h$ in small increments and waiting for transients to decay at each step. To obtain the stationary solution of the amplitude equation we set $\partial \psi / \partial T=0$ in eqn (11.64) and solve it numerically as a boundary value problem over an interval of length $L$, with boundary conditions $\psi(X=0)=\psi(X=L)=0$. We use the approximate expression $\psi_{\text {app }}(X)$ [eqn 11.75 ] as an initial guess. Having identified an upper stability boundary $h=\sqrt{1+\gamma^{2}}$ and an approximate lower existence boundary, given by Eq. (11.74), we must make use of the numerically obtained localized solutions $\psi(X)$ in order to examine the stability within these boundaries [see Kenig et al. $(2009 b)$ for details]. The stability diagram of both the analytical solution $\Psi_{+}$ for $\eta=0$ (Barashenkov et al., 1991) and the numerical solution $\psi(X)$ for $\eta=0.1$ are displayed in Fig. 11.5. Numerical integration of the underlying equations of motion 11.58 confirms the stability analysis, based on the amplitude equation (Kenig et al., 2009b).

Figure 11.5 highlights the effects of nonlinear damping on localized solutions. The first effect is to raise the lower existence boundary. This is explained by the fact that the additional energy lost through nonlinear damping has to be compensated by an increase in the strength of the parametric drive, as predicted by the approximate expression (11.74). The second effect is that nonlinear damping increases the area in the $(h, \gamma)$ parameter space where solitons are stable (blue dots). In particular, the shape of the unstable region for $\eta>0($ red $*$ s) becomes qualitatively different. There are values of $\gamma$ for which an increase in the drive amplitude $h$ initially induces an instability of the soliton, while upon further increase of $h$ the soliton regains its stability. This can be explained by noting that the amplitude of the soliton - given approximately by Eq. (11.72) — increases as $h$ becomes larger, thereby enhancing the effect of nonlinear damping. This increase of damping exerts a similar stabilizing effect as that of increasing $\gamma$ in the absence of nonlinear damping.

\subsubsection{Brief survey of applications}

Kenig et al. (2009b) studies a host of dynamical phenomena using their version of the PDNLS equation (11.64). These include questions concerning the interaction of pairs of solitons, which can be either attractive or repulsive, depending on the relative phase of the two solitons; the possibility of pairs of solitons to form bound states; and 


\section{Collective Dynamics in Arrays of Coupled Nonlinear Resonators}

the ability of a boosted soliton spontaneously to split into two. We urge the reader to consult the original work for additional detail, and only emphasize the exquisite agreement between the predictions made with the amplitude equation (11.64), and numerical simulations of the underlying equations of motion (11.58).

There is one particular question that we wish to address here which deals with the procedure for generating solitons in actual experiments, where one cannot simply introduce an approximate soliton as an initial condition like one does in a simulation. Moreover, it is not obvious how dynamically to form solitons starting with a motionless array of resonators, as one needs to take the system sufficiently far from the basin of attraction of the zero solution $\psi(X)=0$, which is also stable whenever solitons are stable. The most direct procedure for avoiding the zero solution, starting from weak random noise, is to drive the system with $h>\sqrt{1+\gamma^{2}}$, so neither the zero solution nor the soliton solutions are stable. As a consequence, a non-zero pattern develops. Stable solitons can then be formed by lowering the drive amplitude to a value $h<\sqrt{1+\gamma^{2}}$ for which the zero solution and the soliton solutions are both stable, if the non-zero pattern that was obtained is outside the basin of attraction of the zero solution.

This simple procedure, sometimes called self trapping - which could be implemented experimentally in a straightforward manner - is demonstrated in the top panels of Fig. 11.6 showing a numerical simulation of the equations of motion (11.58) with fixed boundary conditions, using $N=199$ resonators. One can see that the initial transient that forms becomes unstable upon lowering the drive amplitude, giving rise to the formation of a number of solitons. Note that before reaching steady state, a pair of solitons merges into one, and another pair attracts and forms a bound state. Both of these effects were studied by Kenig et al. (2009b). The emerging isolated solitons agree well with the approximate analytical form (11.76), determined earlier, with only their central positions $X_{0}$ used as fitting parameters.

A more controlled procedure for generating solitons would be to initiate the array in a particular non-zero state and then to drive it outside its known stability boundaries. This has been considered in the past in systems without nonlinear damping, using the non-zero uniform solution of the PDNLS (Barashenkov et al., 2003). However, it is known for systems with $\eta=0$ that the uniform solution is always unstable against weak modulations and so may be difficult to access dynamically. What Kenig et al. (2009b) discovered was that nonlinear damping can act to stabilize the non-zero spatially-uniform solution, making the procedure for generating solitons through a modulation instability of a uniform state possibly relevant for experiments.

We demonstrate the use of the stable uniform solution in the dynamical formation of solitons in the bottom panels of Fig. 11.6, showing a numerical simulation of the equations of motion 11.58 with periodic boundary conditions, using $N=200$ resonators. The array is initiated with the large-amplitude uniform solution and is driven within the stability boundary of this state, as calculated from the amplitude equation 11.64). After a long time during which the uniform solution indeed remains stable, the drive amplitude is lowered below the stability threshold for this solution, but within the stability boundaries of the soliton solutions, and solitons are formed via a modulation of the unstable uniform state. 


\section{Acknowledgments}

This work was supported by the US-Israel Binational Science Foundation (BSF) through Grant No. 2004339, by the NSF through Grant No. DMR-1003337, and by the German-Israeli Foundation (GIF) through Grant No. 981-185.14/2007. 

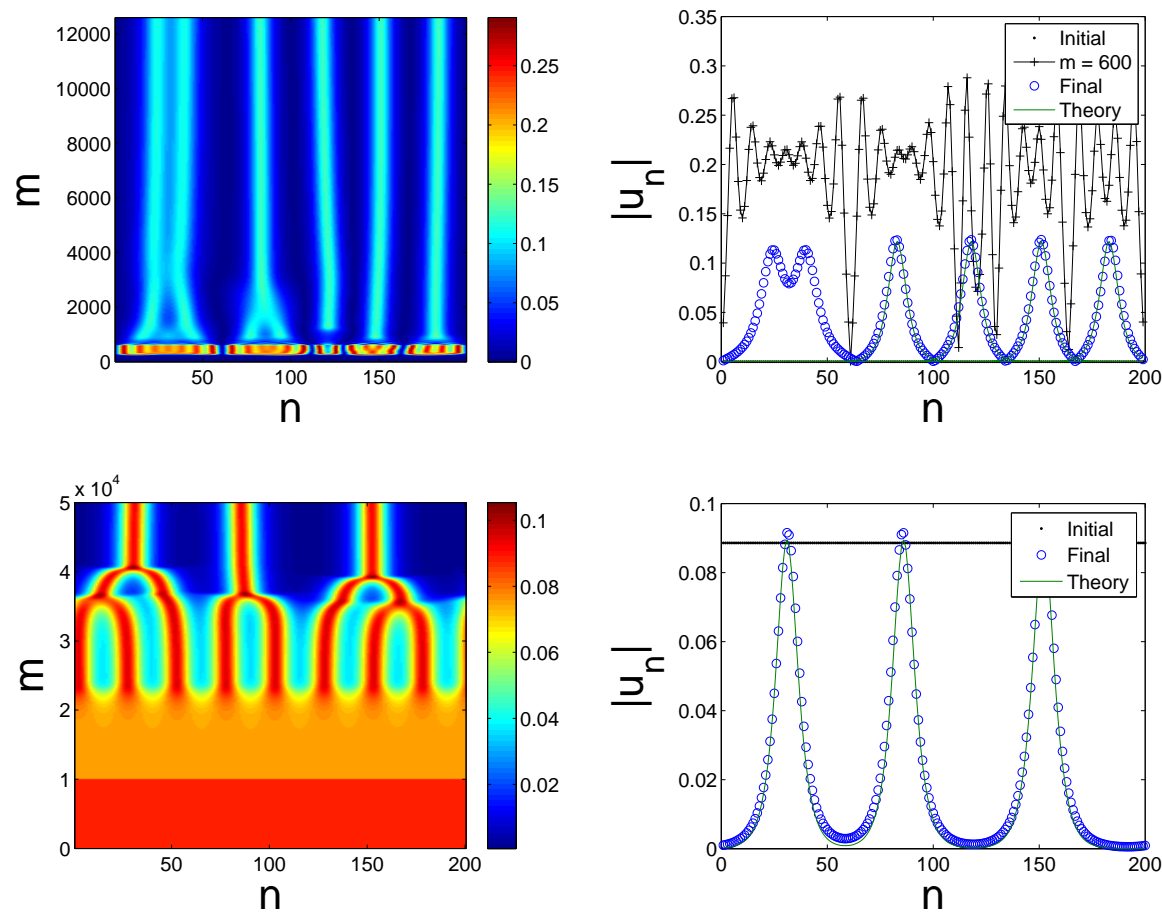

Fig. 11.6 (Color) Numerical simulation of the equations of motion 11.58 showing the dynamical creation of solitons, for $\gamma=1, \eta=0.3, D=0.25, \epsilon=0.01$, and $\omega_{p}=1.002 \omega$. Plotted are the absolute values of the displacements of the resonators, which alternate between positive and negative values. Left panels show the time evolution, with $m$ counting the number of drive periods. Right panels show the initial (black dots) and final (blue circles) states along with the analytical form of the solitons (green solid line), using only their central positions $X_{0}$ as fitting parameters. Top panels: A simulation of 199 resonators with fixed boundary conditions is initiated with random noise and a drive amplitude of $h=5$, which is above the upper stability limit, $h=\sqrt{1+\gamma^{2}}=\sqrt{2}$, for both the zero-state and the solitons. At time $m=600$ drive periods, after a non-zero transient (black $+\mathrm{s}$ in the right panel) has developed, the drive amplitude is lowered to $h=1.35<\sqrt{2}$, yielding stable solitons. Bottom panels: A simulation of 200 resonators with periodic boundary conditions is initiated with the uniform non-zero solution and a drive amplitude of $h=1.3$, which is above the calculated stability threshold, $h_{t h} \simeq 1.26$, for this state (Kenig et al. 2009b). After $m=10000$ drive periods during which the uniform state remains stable, the drive amplitude is lowered to $h=1.2<h_{t h}$, yielding stable solitons. From Kenig et al. (2009b). Copyright (2009) American Physical Society. 


\section{References}

Acebrón, J. A., Bonilla, L. L., Vicente, C. J. P., Ritort, F., and Spigler, R. (2005). Rev. Mod. Phys., 77, 137-184.

Aldridge, J. S. and Cleland, A. N. (2005). Phys. Rev. Lett., 94, 156403.

Alexeeva, N. V., Barashenkov, I. V., and Tsironis, G. P. (2000, Apr). Phys. Rev. Lett., 84(14), 3053-3056.

Barashenkov, I. V., Bogdan, M. M., and Korobov, V.I. (1991). Europhys. Lett, 15, 113-118.

Barashenkov, I. V., Cross, S., and Malomed, Boris A. (2003, Nov). Phys. Rev. E, 68(5), 056605 .

Bennett, M., Schatz, M. F., Rockwood, H., and Wiesenfeld, K. (2002). Proc. Roy. Soc. Series A, 458, 563.

Binder, P., Abraimov, D., Ustinov, A. V., Flach, S., and Zolotaryuk, Y. (2000). Phys. Rev. Lett., 84, 745-748.

Blasius, Bernd and Tönjes, Ralf (2005). Phys. Rev. Lett., 95, 084101.

Bromberg, Yaron (2004). Master's thesis, Tel Aviv University.

Bromberg, Yaron, Cross, M. C., and Lifshitz, Ron (2006). Phys. Rev. E, 73, 016214.

Buks, E. and Roukes, M. L. (2002). J. Microelectromech. Syst., 11, 802-807.

Buks, Eyal and Yurke, Bernard (2006). Phys. Rev. E, 74, 046619.

Burke, J., Yochelis, A., and Knobloch, E. (2008). SIAM J. Applied Dynamical Systems, 7, 651-711.

Campbell, David K., Flach, Sergej, and Kivshar, Yuri S. (2004). Physics Today, $\mathbf{5 7}(1), 43-49$.

Chen, Wei-Zhong (1994, Jun). Phys. Rev. B, 49(21), 15063-15066.

Cheskis, D., Bar-Ad, S., Morandotti, R., Aitchison, J. S., Eisenberg, H. S., Silberberg, Y., and Ross, D. (2003). Phys. Rev. Lett., 91(22), 223901.

Cleland, Andrew (2003). Foundations of Nanomechanics. Springer, Berlin.

Cleland, A. N. and Geller, M. R. (2004). Phys. Rev. Lett., 93(7), 070501.

Craighead, Harold G. (2000). Science, 290, 1532.

Cross, Michael and Greenside, Henry (2009). Pattern Formation and Dynamics in Nonequilibrium Systems. Cambridge University Press, Cambridge.

Cross, M. C. and Hohenberg, P. C. (1993). Rev. Mod. Phys., 65, 851-1112.

Cross, M. C., Rogers, J. L., Lifshitz, Ron, and Zumdieck, A. (2006). Phys. Rev. E, 73, 036205 .

Cross, M. C., Zumdieck, A., Lifshitz, Ron, and Rogers, J. L. (2004). Phys. Rev. Lett., 93, 224101.

DeMartini, B.E., Rhoads, J.F., Turner, K.L., Shaw, S.W., and Moehlis, J. (2007). J. Microelectromech. Syst., 16, 310-318.

Denardo, Bruce, Galvin, Brian, Greenfield, Alan, Larraza, Andrés, Putterman, Seth, 
and Wright, William (1992, Mar). Phys. Rev. Lett., 68(11), 1730-1733.

Dror, N. and Malomed, B. A. (2009). Phys. Rev. E, 79, 016605.

Dykman, M. I. and Krivoglaz, M. A. (1975). Physica Status Solidi (b), 68, 111-123.

Dykman, M. I. and Krivoglaz, M. A. (1984). In Physics Reviews (ed. I. M. Khalatnikov), Volume 5, Soviet Scientific Reviews, Section A, pp. 265-441. Harwood Academic, New York.

Eisenberg, H. S., Morandotti, R., Silberberg, Y., Bar-Ad, S., Ross, D., and Aitchison, J. S. (2001). Phys. Rev. Lett., 87, 043902.

Eisenberg, H. S., Silberberg, Y., Morandotti, R., Boyd, A. R., and Aitchison, J. S. (1998, Oct). Phys. Rev. Lett., 81(16), 3383-3386.

Ekinci, K. L., Huang, X. M. H., and Roukes, M. L. (2004). App. Phys. Lett., 84(22), 4469-4471.

Ezerskiı̌, A. B., Rabinovich, M. I., Reutov, V. P., and Starobinets, I. M. (1986). Zh. Eksp. Teor. Fiz., 91, 2070-2083. [Sov. Phys. JETP 64, 1228 (1986)].

Feng, X. L., White, C. J., Hajimiri, A., and Roukes, Michael L. (2008). Nat. Nano., 3, $342-346$.

Fleischer, Jason W., Segev, Mordechai, Efremidis, Nikolaos K., and Christodoulides, Demetrios N. (2003). Nature, 422, 147-150.

Greywall, D. S., Yurke, B., Busch, P. A., Pargellis, A. N., and Willett, R. L. (1994, May). Phys. Rev. Lett., 72(19), 2992-2995.

Huang, X. M. H., Zorman, C. A., Mehregany, M., and Roukes, Michael L. (2003). Nature, 421, 496.

Ilic, B., Craighead, H. G., Krylov, S., Senaratne, W., Ober, C., and Neuzil, P. (2004). J. Appl. Phys., 95, 3694.

Kacem, N., Arcamone, J., Perez-Murano, F., and Hentz, S. (2010). J. Micromech. Microeng., 20, 045023.

Kacem, N., Hentz, S., Pinto, D., Reig, B., and Nguyen, V. (2009). Nanotechnology, 20, 275501.

Karabalin, R. B., Cross, M. C., and Roukes, M. L. (2009). Phys. Rev. B, 79(16), 165309.

Karabalin, R. B., Lifshitz, Ron, Cross, M. C., Matheny, M. H., Masmanidis, S. C., and Roukes, M. L. (2011). Phys. Rev. Lett., 106, 094102.

Katz, Itamar, Lifshitz, Ron, Retzker, Alex, and Straub, Raphael (2008). New J. Phys., 10, 125023.

Katz, Itamar, Retzker, Alex, Straub, Raphael, and Lifshitz, Ron (2007). Phys. Rev. Lett., 99, 040404.

Kenig, Eyal, Lifshitz, Ron, and Cross, M. C. (2009a). Phys. Rev. E, 79, 026203.

Kenig, Eyal, Malomed, Boris A., Cross, M. C., and Lifshitz, Ron (2009b). Phys. Rev. $E, \mathbf{8 0}(4), 046202$.

Kenig, Eyal, Tsarin, Yuriy A., and Lifshitz, Ron (2011). Phys. Rev. E, 84, 016212. Kozinsky, I., Postma, H. W. Ch., Kogan, O., Husain, A., and Roukes, M. L. (2007). Phys. Rev. Lett., 99, 207201.

Kramer, L., Schober, H. R., and Zimmermann, W. (1988). Physica D, 31, 212-226. Kuramoto, Y. (1975). Lect. Notes Phys., 39, 420.

LaHaye, M. D., Buu, O., Camarota, B., and Schwab, K. C. (2004). Science, 304, 
$74-77$.

Lee, Jungchul, Shen, Wenjiang, Payer, Kris, Burg, Thomas P., and Manalis, Scott R. (2010). Nano. Lett., 10, 2537-2542.

Li, Mo, Tang, Hong X., and Roukes, Michael L. (2007). Nature Nanotechnology, 2, $114-120$.

Lifshitz, Ron and Cross, M. C. (2003). Phys. Rev. B, 67, 134302.

Lifshitz, Ron and Cross, M. C. (2008). In Review of Nonlinear Dynamics and Complexity (ed. H. G. Schuster), Volume 1, pp. 1-52. Wiley-VCH, Weinheim.

Lifshitz, Ron and Cross, M. C. (2010). In Nonlinear Dynamics of Nanosystems (ed. G. Radons, B. Rumpf, and H. G. Schuster), Chapter 8. Wiley-VCH, Weinheim.

Longhi, Stefano (1996, May). Phys. Rev. E, 53(5), 5520-5522.

Maniadis, P. and Flach, S. (2006). Europhys. Lett., 74, 452-458.

Matthews, P. C., Mirollo, R. E., and Strogatz, S. H. (1991). Physica D, 52, 293.

Miao, Guoqing and Wei, Rongjue (1999, Apr). Phys. Rev. E, 59(4), 4075-4078.

Milner, S. T. (1991). J. Fluid Mech., 225, 81-100.

Naik, A., Buu, O., LaHaye, M. D., Armour, A. D., Clerk, A. A., Blencowe, M. P., and Schwab, K. C. (2006). Nature, 443, 193-196.

Naik, A. K., Hanay, M. S., Hiebert, W. K., Feng, X. L., and Roukes, Michael L. (2009). Nature Nanotechnology, 4, 445-450.

O'Connell, A. D., Hofheinz, M., Ansmann, M., Bialczak, Radoslaw C., Lenander, M., Lucero, Erik, Neeley, M., Sank, D., Wang, H., Weides, M., Wenner, J., Martinis, John M., and Cleland, A. N. (2010). Nature, 464, 697-703.

Ovchinnikov, A. A. and Érikhman, N. S. (1982). Sov. Phys. Usp., 25, 738-755.

Rhoads, Jeffrey F., Shaw, Steven W., and Turner, Kimberly L. (2010). J. Dyn. Sys. Meas. Control, 132, 034001.

Riecke, H. (1990). Europhys. Lett., 11, 213-218.

Rocheleau, T., Ndukum, T., Macklin, C., Hertzberg, J. B., Clerk, A. A., and Schwab, K. C. (2010). Nature, 463, 72-75.

Roukes, Michael L. (2001). Scientific American, 285, 42-49.

Rugar, D., Budakian, R., Mamin, H. J., and Chui, B. W. (2004). Nature, 430 , 329-332.

Sakaguchi, H. (1988). Prog. Theor. Phys., 79, 39-46.

Sakaguchi, Hidetsugu, Shinomoto, Shigeru, and Kuramoto, Yoshiki (1988). Prog. Theor. Phys., 79, 1069-1079.

Sánchez-Morcillo, V. J., Pérez-Arjona, I., Silva, F., de Valcárcel, G. J., and Roldán, E. (2000). Opt. Lett., 25(13), 957-959.

Sato, M., Hubbard, B. E., English, L. Q., Sievers, A. J., Ilic, B., Czaplewski, D. A., and Craighead, H. G. (2003a). Chaos, 13(2), 702-715.

Sato, M., Hubbard, B. E., and Sievers, A. J. (2006). Revs. Mod. Phys., 78, 137.

Sato, M., Hubbard, B. E., Sievers, A. J., Ilic, B., and Craighead, Harold G. (2004). Europhys. Lett., 66, 318-323.

Sato, M., Hubbard, B. E., Sievers, A. J., Ilic, B., Czaplewski, D. A., and Craighead, H. G. (2003b). Phys. Rev. Lett., 90(4), 044102.

Sato, M. and Sievers, A. J. (2004). Nature, 432, 486-488.

Sato, M. and Sievers, A. J. (2007). Phys. Rev. Lett., 98(21), 214101. 
Sato, M. and Sievers, A. J. (2008). Low Temp. Phys., 34(7), 543-548.

Scheible, Dominik V., Erbe, Artur, Blick, Robert H., and Corso, Gilberto (2002). App. Phys. Lett., 81(10), 1884-1886.

Schwab, K., Henriksen, E. A., Worlock, J. M., and Roukes, M. L. (2000). Nature, 404, 974-977.

Schwarz, U. T., English, L. Q., and Sievers, A. J. (1999). Phys. Rev. Lett., 83, 223-226.

Sievers, A. J. and Takeno, S. (1988, Aug). Phys. Rev. Lett., 61(8), 970-973.

Swanson, B. I., Brozik, J. A., Love, S. P., Strouse, G. F., Shreve, A. P., Bishop, A. R., Wang, W.-Z., and Salkola, M. I. (1999). Phys. Rev. Lett., 82, 3288-3291.

Trías, E., Mazo, J. J., and Orlando, T. P. (2000, Jan). Phys. Rev. Lett., 84(4), $741-744$.

Turner, Kimberly L., Miller, Scott A., Hartwell, Peter G., MacDonald, Noel C., Strogatz, Steven H., and Adams, Scott G. (1998). Nature, 396, 149-152.

Wang, Xinlong and Wei, Rongjue (1997, Apr). Phys. Rev. Lett., 78(14), 2744-2747.

Wang, Xinlong and Wei, Rongjue (1998, Feb). Phys. Rev. E, 57(2), 2405-2410.

Weig, E. M., Blick, R. H., Brandes, T., Kirschbaum, J., Wegscheider, W., Bichler, M., and Kotthaus, J. P. (2004). Phys. Rev. Lett., 92, 046804.

Weinstein, Dana and Bhave, Sunil A. (2010). Nano Lett., 10, 1234-1237.

Winfree, A. T. (1967). J. Theor. Bio., 16, 15.

Yang, Y. T., Callegari, C., Feng, X. L., Ekinci, K. L., and Roukes, M. L. (2006). Nano. Lett., 6, 583-586.

Zaitsev, Stav, Almog, Ronen, Shtempluck, Oleg, and Buks, Eyal (2005). In Proccedings of the 2005 International Conference on MEMS, NANO, and Smart Systems (ICMENS 2005), pp. 387-391. IEEE Computer Society.

Zhang, Wenhua, Baskaran, Rajashree, and Turner, Kimberly L. (2002). Sensors and Actuators A, 102, 139-150.

Zhang, Wenbin and Viñals, Jorge (1995, Jan). Phys. Rev. Lett., 74(5), 690-693. 


\section{Index}

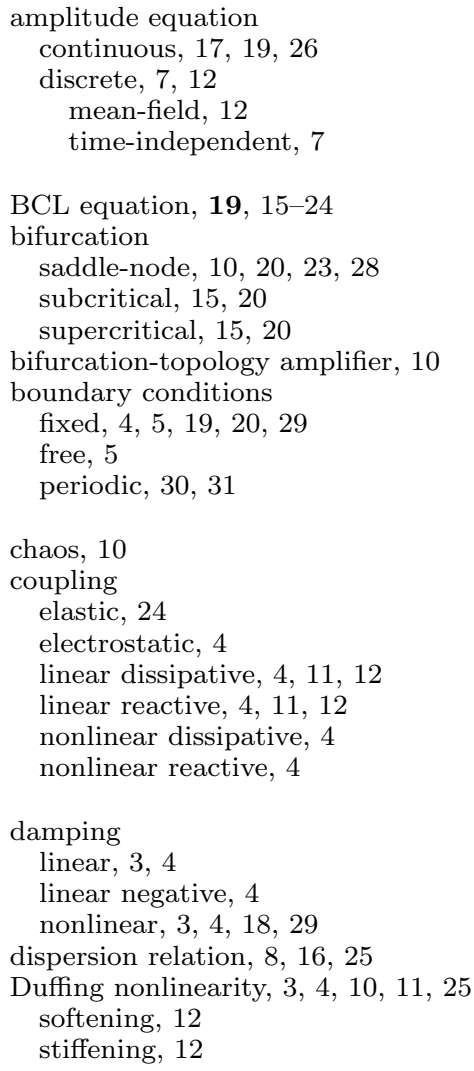

Eckhaus instability, 20, 21, 23 equation of motion one-dimensional array, 4 scaled, 5, 11, 16, 25

single resonator, 3 scaled, 3

frequency pulling, 10-12, 25

Ginzburg-Landau equation, 26 group velocity, 18, 25

homoclinic orbits, 10

Huygens clocks, 10 hysteresis, 8, 9, 14

Kuramoto model, 13

localized modes, 24-31

Melnikov, 10

multiple scales, 5

ansatz, 6, 11, 16, 18, 25

multistability, 9, 10, 14, 15, 22

neutral stability curve, 20,21

nonlinear saturation, 4, 11

nonlinear Schrödinger equation

parametrically driven, 26, 24-31

with linear damping, 27

with nonlinear damping, 26

parametric drive, 3

pattern selection, 21

PDNLS equation, 26, 24-31

quasistatic sweep

of coupling strength, 14

of drive amplitude, 21, 22, 29

of drive frequency, 9,10

rotating frame, 6

secular perturbation theory, 3

secular term, 6, 12, 17, 19, 25

single-mode extended solution, 19 scaled, 19

stability, 20-21

soliton solution, 27-31

approximate with nonlinear damping, 29

modulation instability of uniform state, 31 self trapping, 31

solvability condition, $7,12,17,19,25$

stability diagram, 14-16, 21, 22, 27

steady-state solution, 7, 19, 29

scaled, $7,19,27$

synchronization, 10-15

order parameter, 12, 13

umklapp condition, 7

van der Pohl oscillator, 4, 11 